\title{
COCRIAÇÃo E A COPRODUÇÃO GERAM VALOR E SATISFAÇÃo AO CLIENTE? ANÁLISE DA PERCEPÇÃO DE CLIENTES DE
INSTITUIÇÕES FINANCEIRAS
}

\author{
${ }^{1}$ Fábio Rogério de Morais \\ 2 Edgar de Souza Pandolfi \\ ${ }^{3}$ Luciana Trindade Sanagioto
}

Objetivo do estudo: O objetivo deste estudo foi analisar os efeitos da coprodução e da cocriação na cocriação de valor e na satisfação do consumidor de serviços financeiros.

Metodologia/Abordagem: Utilizou-se de modelagem de equações estruturais (structural equations modeling - SEM) para a avaliação empírica do modelo teórico que investiga o valor resultante da interação na produção ou criação conjunta e se o engajamento na cocriação e na coprodução possui efeitos positivos na construção do valor e da satisfação.

Principais resultados: $\mathrm{O}$ modelo apresentou estatísticas próximas dos valores aceitáveis, porém não suficientes para afirmar que há efeito da coprodução e da cocriação sobre o valor cocriado e a satisfação do cliente. É possível afirmar que há correlação entre as variáveis de coprodução e de Cocriação, mas o baixo poder de explicação aponta para necessidade de ajuste no modelo ou revisão dos constructos.

Contribuições teóricas/metodológicas: Ressalta-se a relação entre coprodução e cocriação, pois o modelo amplia o conhecimento sobre os constructos da coprodução e da cocriação aplicadas ao contexto financeiro, com categorias e proposta de modelagem, ao refinar as ideias de coprodução, cocriação, cocriação de valor e valor percebido (LDS).

Relevância/originalidade: $O$ estudo constrói parâmetros de medição do valor cocriado na cocriação e coprodução de serviços e indica que os clientes de serviços financeiros coproduzem ou cocriam na extensão do fornecedor, e não do cliente.

Palavras-chave: Cocriação e Coprodução. Cocriação de Valor. Satisfação. Serviços Financeiros.

\section{Como citar:}

Morais, F. R., Pandolfi, E.S. de, \& Sanagioto, L. T. (2020). A cocriação e a coprodução geram valor e satisfação ao cliente? Análise da percepção de clientes de instituições financeiras. Revista Brasileira de Marketing, 19(1), 126-149. https://doi.org/10.5585/remark.v19i1.17141.

Universidade Federal de Rondônia - UNIR, Rondônia, (Brasil). fabiomorais@unir.br

${ }^{2}$ Faculdade de Educação e Meio Ambiente - FAEMA, Rondônia, (Brasil). edgarpandolfi@gmail.com

3 (D) Faculdades Associadas de Ariquemes - FAAr, Rondônia, (Brasil). luciana.sanagioto@hotmail.com 


\section{Introdução}

A cocriação e a coprodução de serviço se desenvolvem como um novo paradigma na literatura de gestão de negócios, na qual se evidencia a construção do valor relacional, em contraposição às teorias econômicas de valor derivado exclusivamente da transação (Galvagno \& Dalli, 2014; Pohlmann \& Kaartemo, 2017). A cocriação é a participação do cliente no processo colaborativo de produzir valor material ou simbólico (Galvagno \& Dalli, 2014; Gronröos, 2009; Lusch \& Vargo, 2006), enquanto a coprodução envolve o cliente na produção de bens e serviços, ao executar toda ou parte da produção com o fornecedor (Agrawal \& Rahman, 2015; Ostrom, 1996). Tal proposição destaca que há uma sequência de necessidades dos consumidores em estabelecer relacionamentos e vivenciar experiências de valor nas trocas econômicas (Prahalad \& Ramaswamy, 2004), e dos fornecedores em compartilhar a produção com os clientes (Etgar, 2008). Assim, a proposição da Lógica Dominante de Serviço (LDS) é de que o valor resulta da interação em processos conjuntos entre os clientes e os fornecedores, na produção ou criação conjunta de produtos e serviços (Vargo \& Lusch, 2004).

Embora a literatura destaque que o valor e a satisfação do consumidor ao transacionar (Williams \& Naumann, 2011) é construído no relacionamento capaz de gerar experiências de valor nos processos de trocas econômicas (Prahalad \& Ramaswamy, 2004), é necessário avançar no conhecimento sobre como e onde o valor é cocriado nos processos de coprodução e cocriação de serviços. Há evidencias de que a existência de relacionamento, por si, é insuficiente para gerar o valor e a satisfação do cliente coprodutor ou cocriador. A LDS sugere que a interação e o engajamento do cliente na cocriação e na coprodução de produtos e serviços possui efeitos positivos na construção da experiência de valor conjunto (Prahalad \& Ramaswamy, 2000, 2004; Vargo \& Lusch, 2004) e na satisfação do consumidor (Williams \& Naumann, 2011). Nota-se, ainda, que a interação e o engajamento entre os clientes e os fornecedores em processos coproduzidos ou cocriados melhoram a eficiência produtiva, a redução de custos em longo prazo, e a qualidade dos serviços prestados (Alford, 2002; Bendapudi \& Leone, 2003; Gronröos, 2009, 2011; Morais \& Santos, 2015; Ramirez, 1999), e contribui para o maior retorno financeiro às organizações (Williams \& Naumann, 2011).

A LDS (Vargo \& Lusch, 2006) diferencia a coprodução da cocriação, por meio do papel do cliente no processo de criação de valor conjunto. A coprodução vê o consumidor como um agente passivo e concentra-se no modo como a organização pode obter benefícios produtivos com o envolvimento do consumidor na produção, enquanto a cocriação classifica o cliente como agente ativo na relação com a organização (Prahalad \& Ramaswamy, 2004; Vargo \& Lusch, 2004). Esta proposta de Vargo e Lusch (2004) é destaque na literatura de gestão e no meio corporativo, com 
trajetória evolutiva nas áreas de cocriação de valor, integração de recursos, marcas e inovação (Pohlmann \& Kaartemo, 2017). No entanto, há críticas à LDS, especialmente quanto à visão metafórica da cocriação de valor (Gronröos \& Voima, 2013) e à ausência de métricas para o dimensionamento empírico do valor decorrente das práticas de cocriação e de coprodução (Morais \& Santos, 2015). Os resultados de pesquisas anteriores indicam que a comunidade científica tem intensificado a avaliação sobre as questões ontológicas e epistemológicas fundamentais da LDS, como, por exemplo, os fenômenos complexos, as conceituações genéricas, os processos de democratização e as práticas na institucionalização (Pohlmann \& Kaartemo, 2017).

Neste contexto, esta pesquisa apoiou-se nos estudos de Ramirez (1999), Vargo e Lusch (2004), Etgar (2008), Gronröos (2009), Grönroos (2011), e Morais e Santos (2015), e buscou identificar os efeitos da cocriação e da coprodução sobre o valor cocriado, e a satisfação de usuários de serviços financeiros, caracterizada como avaliação de práticas institucionalizadas (Pohlmann \& Kaartemo, 2017). A proposição é que os consumidores em ambientes de serviços são fornecedores de recursos e participantes na criação de valor (Prahalad \& Ramaswamy, 2004; Vargo \& Lusch, 2004), e a cocriação e a coprodução aumentam o valor percebido e a satisfação. Para isso, avaliaramse as características e os resultados das interações cotidianas em agências físicas ou em outros ambientes de atendimento, determinantes para a cocriação de valor e a satisfação do consumidor (Parasuraman, Zeithaml, \& Berry, 1985), no caso, de serviços financeiros. Portanto, buscou-se responder a seguinte pergunta a de pesquisa: quais os efeitos da cocriação e da coprodução de serviços, na percepção de valor cocriado e na satisfação percebida por consumidores, em serviços financeiros?

A escolha do setor financeiro como contexto de análise deve-se a sua consideração, na literatura, como área relevante para a coprodução e cocriação de serviços. Auh, Bell, McLeod, \& Shih (2007) destacam que há relação entre a coprodução e a lealdade do cliente do sistema financeiro. Acredita-se que a interação entre o consumidor e a empresa de serviços financeiros favorece a criação ou a produção conjunta de bens de consumo, e possibilita a criação conjunta de valor, de acordo com as particularidades dos clientes (Prahalad \& Ramaswamy, 2004). Pesquisas destacam que as instituições financeiras passam por contínua reestruturação de processos produtivos, e enfatizam a melhoria dos serviços prestados para atender às necessidades dos clientes (Arasli, Mehtap-Smadi, \& Katircioglu, 2005). Dessa forma, os relacionamentos mutuamente benéficos entre o consumidor e a organização permitem a troca de experiências e, consequentemente, promovem a criação de valor, tanto na cocriação quanto na coprodução (Morais \& Santos, 2015).

Ribeiro, Machado e Tinoco (2010) afirmam que a satisfação e a lealdade dos clientes aumentam a lucratividade das instituições financeiras, mas os fatores ligados à cocriação de valor e 
sua influência na satisfação precisam ser examinados. Assim, a amostragem da pesquisa foi composta por clientes bancários e clientes de cooperativas de crédito. Embora os clientes associados às cooperativas de créditos apresentem características diferentes dos clientes bancários, pois, além de usar os produtos, têm participação no capital institucional, e, por isso, acredita-se que estejam mais predispostos à cocriação (Duarte, Moreira, Ferraresi, \& Gerhard, 2016), poucas evidências teóricas capazes de justificar estas diferenças foram identificadas na literatura. Assim, optou-se por avaliá-los no conjunto amostral que aborda a ação da cocriação e de coprodução, de modo separado, mas engloba-os nos resultados de valor e de satisfação percebidos pelos clientes destes serviços.

\section{0 modelo e desenvolvimento das hipóteses}

Este estudo evoluiu do modelo teórico proposto por Morais e Santos (2015), em que os autores mostram que a cocriação de valor é o elemento resultante da interação e do engajamento do cliente no processo de criação e produção conjunta de produtos e serviços (Figura 1). O modelo expõe que o enfoque no fornecedor e na produção se concentram no eixo $\boldsymbol{x}$, onde $\boldsymbol{x}$ é a matriz de delineamento dos efeitos fixos, de dimensões $n \times p$; o cliente e o uso ou consumo estão no eixo $\mathbf{y}$, em que y é um vetor de variáveis observáveis, de dimensões $n \times 1$; e $z$ é derivado da cocriação de valor resultante da interação entre $\boldsymbol{x}$ ou $\boldsymbol{y}$, onde z é a matriz de incidência de efeitos aleatórios (covariáveis), de dimensões $n \times q$. Para Morais e Santos (2015), a interação entre os eixos $(\boldsymbol{x})$ e $(\boldsymbol{y})$ poderá apresentar variação no valor cocriado no vértice do eixo $(z)$, de acordo com as determinantes da interação na relação econômica entre cliente e fornecedor.

Orientado pelas lacunas teóricas identificadas, o objetivo geral desta pesquisa é estudar o efeito da cocriação e da coprodução (fornecedor/consumidor) de serviços, na percepção de valor cocriado e de satisfação percebida pelo consumidor, em serviços financeiros. Assim, o modelo apresenta que, quanto maior a interação entre o cliente engajado no processo de cocriação ou coprodução e o fornecedor, maior será o valor cocriado percebido (Morais \& Santos, 2015). Acrescenta-se nesta análise empírica a dimensão satisfação do consumidor, com o objetivo de identificar se a cocriação e a coprodução impactam na satisfação do cliente. 
Figura 1- Modelo teórico de análise

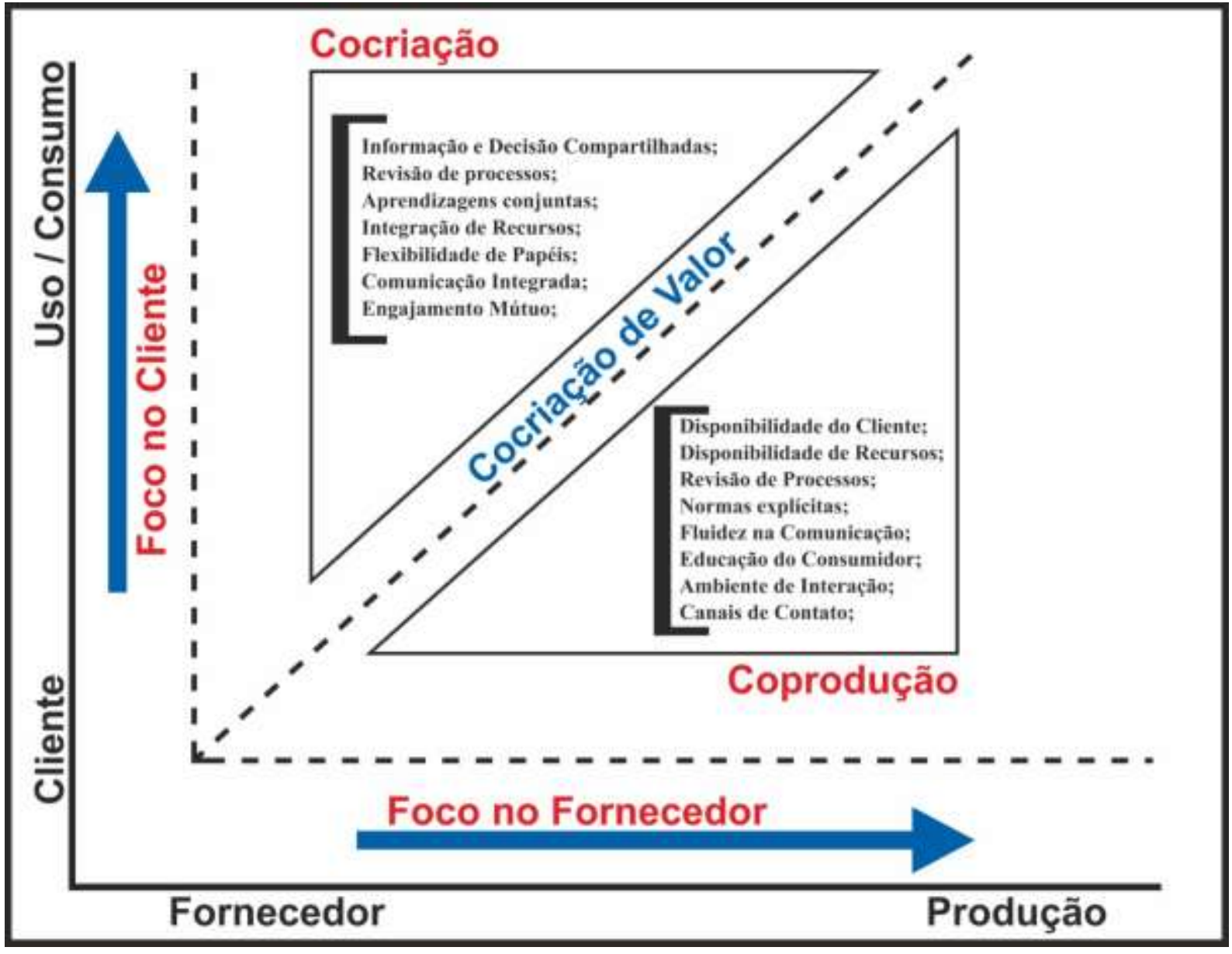

Fonte: Morais e Santos (2015, p. 245).

Neste estudo, usou-se a modelagem de equações estruturais (structural equations modeling - SEM) como técnica confirmatória, em que o modelo foi desenvolvido antes da coleta dos dados, e o objetivo foi verificar se os dados coletados confirmariam ou não o modelo teórico. A SEM é compreendida como um método de pesquisa que envolve atividades e decisões sobre a escolha do referencial teórico, elaboração do modelo estrutural e do modelo de mensuração (Hair et al., 2009).

A SEM permite testes confirmatórios da estrutura psicométrica de escalas de medida e também analisar relações explicativas entre múltiplas variáveis, simultaneamente, sejam essas latentes ou observadas (Hair et al., 2009). Desse modo, foi adotada a SEM para analisar mais de uma variável dependente, onde $\boldsymbol{x}$ influencia $\boldsymbol{y}$ e $\boldsymbol{y}$ influencia $\boldsymbol{z}$, pois serviços são inerentemente cocriados e/ou coproduzidos (Chathoth, Altinay, Harrington, Okumus, \& Chan, 2013) em esferas distintas da interação entre clientes e fornecedores (Morais \& Santos, 2015). A justificativa para o uso da SEM é que se pode obter modelos causais compatíveis com os dados, e confirmar a alternativa que tem maior apoio da teoria. Esta técnica possibilita a investigação de quão bem as variáveis preditoras explicam as variáveis dependentes, e quais variáveis preditoras são mais 
importantes na explicação do modelo (Hair et al., 2009). Para atingir a qualidade metodológica com a modelagem de equações estruturais, utilizam-se os parâmetros estabelecidos por Bido, Souza, Silva, Godoy e Torres (2012), em que os autores apresentam as etapas adequadas para preparação e uso da SEM. A seguir, este estudo apresenta as hipóteses fundamentadas na teoria e o modelo estrutural proposto, com a direção esperada para os parâmetros estabelecidos para a confirmação.

\subsection{Desenvolvimento das hipóteses}

A cocriação acontece quando o cliente é parte do processo de criação de produtos e serviços, desenvolvido no relacionamento com a organização (Gronröos, 2009). Assim, permite que diferentes atores participem e contribuam para o processo de criação em trocas econômicas, por meio de competências e conhecimentos compartilhados (Vargo \& Lusch, 2004), que resultem em bens de uso e consumo que atendam às necessidades das partes (Grönroos, 2011). Por outro lado, a coprodução é a participação do cliente ao trabalhar dentro de parâmetros pré-estipulados pelo fornecedor, ao fazer todo ou parte do processo produtivo (Etgar, 2008; Ostrom, 1996; Ojasalo, 2010) em uma indústria (serviços/produtos).

Vê-se que a coprodução e a cocriação são desenvolvidas em bases processuais e conceituais diferentes de engajamento (Morais \& Bonomi, 2015) para o acesso a recursos que atores envolvidos acreditam ter potencial de valor (Chandler \& Vargo, 2011). As duas relações, embora distintas, se desenvolvem em plataformas relacionais similares, exceto pelo modus operandi da ação dos players envolvidos na construção do resultado de valor. A cocriação tem os seus processos centrados no cliente (Gronröos, 2009), enquanto a coprodução tem enfoque no fornecedor (Ramirez, 1999; Wu, Wang \&Rouyer, 2019), porém, a teoria de gestão que aborda a construção de valor por meio de ambas, não as apresenta de modo oponentes, entre si. É possível que a coprodução realizada para produzir determinado produto ou serviço possibilite a evolução do engajamento para a cocriação, que pode ocorrer com pequenas alterações nas bases produtivas daquilo que é coproduzido (produto/serviço), como exemplo, o surgimento de novas utilidades para o produto, ou a cocriação de novos processos que favoreçam o desempenho produtivos, e, ainda, a inserção de determinado produto/serviço coproduzido em novos mercados cocriados com os consumidores, especialmente por meio digital (Nambisan \& Baron, 2007, 2009).

Isto reforça a ideia de que, embora a coprodução seja uma atividade centrada na esfera do fornecedor (Gronröos, 2011), ela pode exigir ações que excedam o ato produtivo conjunto (fornecedor/consumidor) e, assim, evoluir para a cocriação de novos processos produtivos, de mercados de consumo ou de utilidade para o que é coproduzido (Ramirez, 1999; Wu, Wang \&Rouyer, 2019). Logo, parece que a transição da coprodução para a cocriação pode acontecer por meio da ampliação de atribuições aos consumidores participantes de processos produtivo conjuntos 
entre fornecedores e consumidores, de modo que, tanto produtos e processos como mercados, podem ser ampliados ou melhorados, cuja suposição teórica é de efeito positivo da coprodução sobre a cocriação (Chandler \& Vargo, 2011). Assim,

H1: a ação de coprodução com fornecedores pode evoluir para a cocriação em serviços, segundo a percepção dos consumidores.

Além disso, a experiência de valor com a coprodução e a cocriação se insere na esfera do relacionamento (Prahalad \& Ramaswamy, 2004). É na interação do fornecedor com o cliente, ao engajá-lo no processo de criação e de produção, que ocorre a influência sobre o valor cocriado (Grönroos \& Voima, 2013; Morais \& Santos, 2015). O valor cocriado decorre da influência mútua entre os players, e pode ocorrer de forma direta, em áreas físicas de contato, ou por meios virtuais, com mecanismos tecnológicos de engajamento na cocriação e coprodução (Nambisan \& Baron, 2007, 2009).

Payne, Storbacka e Frow (2008) afirmam que a interação ao coproduzir ou cocriar pode gerar valor para o cliente. No entanto, se a coprodução classifica o consumidor como um agente passivo e foca nos benefícios produtivos proporcionados ao cliente com o seu envolvimento na produção, enquanto a cocriação considera o cliente como agente ativo na relação com a organização (Prahalad \& Ramaswamy, 2004; Vargo \& Lusch, 2004), ao atuar como parte criativa do serviço a ser ofertado, deve haver diferenças em resultados de valor percebido, ao se comparar a percepção do consumidor. Assim, são duas bases de cocriação de valor, que podem gerar ao consumidor percepções diferentes de valor cocriado (Morais \& Santos, 20015; Vargo \& Lusch, 2004; Gronröos, 2009; Grönroos, 2011).

A análise ancora-se no contexto em que o é valor cocriado, decorrente da orientação do processo de construção do valor (multinível) (Chandler \& Vargo, 2011): horizontais, onde as atividades são coordenadas na coprodução, e verticais, em que a cocriação exige a integração dos parceiros. Isso pode gerar percepções distintas de valor cocriado e apropriado, ainda que ambas as abordagens se sustentam na interação e no relacionamento (Grönroos, 2011).

Há evidências de que a integração do fornecedor com o consumidor melhora o desempenho do Processo de Desenvolvimento de Produtos, com redução de tempo e produtos mais adequados ao mercado (Prandelli et al., 2006). Há, ainda, comprovação dos efeitos positivos da cocriação na avaliação do produto final (Yin, Chan, \& Lam, 2012). No entanto, está ausente nos estudos citados a análise do valor decorrente da ação conjunta na cocriação. Do mesmo modo ocorre na ação denominada coprodução (Ramirez, 1999; Vargo \& Lush, 2004), cuja decisão também é incorporar as competências do consumidor. De acordo com a proposta desta pesquisa, o efeito da cocriação e 
da coprodução tem impacto na percepção de consumidores quanto ao valor cocriado. Acredita-se que a ação de participação (cocriação e coprodução) construa um senso de colaboração e engajamento (Thompson \& Malaviya, 2013) que leve o consumidor a percepção de valor conjunto. Por isso, o ato de cocriar ou coproduzir com o consumidor pode ter efeito sobre a sua percepção de valor. Especificamente,

H2a: a coprodução de serviços é dependente do contexto produtivo e do engajamento dos consumidores, motivo pelo qual o efeito sobre a percepção de valor cocriado é insignificante na coprodução de serviços com baixo engajamento.

H2b: a cocriação de serviços exige maior engajamento com consumidores $e$, por isso, tem efeito positivo sobre a percepção de valor cocriado.

De forma complementar, ressalta-se que a oferta relacional e a interação entre as partes possuem níveis diferentes de engajamento, pois alguns consumidores se permitem engajar e outros não (Morais \& Santos, 2015). Há, ainda, a influência de dimensões do fornecedor que inibem ou facilitam a interação no relacionamento com os clientes. Assim, o valor cocriado se diferencia no modo como a interação e o engajamento de clientes se processa.

A coprodução pode aumentar a satisfação do cliente (Prahalad \& Ramaswamy, 2004), mas o enfoque é na performance financeira da organização, com a transferência total ou parcial da produção (Williams \& Naumann, 2011). A cocriação pode aumentar a satisfação do cliente (Gronröos, 2009), mas o enfoque é no produto da organização, com a transferência total ou parcial do bem ao consumidor (Prandelli et al., 2006; Yin, Chan, \& Lam, 2012). Nesse contexto, Ramirez (1999) afirma que a percepção do consumidor é determinada pelas experiências vividas, portanto, a forma como o valor é cocriado pode melhorar a satisfação do consumidor (Kauffmann \& Marchetti, 2008). De acordo com Zeithaml, Bitner e Gremler (2006), a percepção do cliente é o que determina a satisfação ou não, em serviços.

Os processos de cocriação e de coprodução têm diferenças no modo de interação e no grau de engajamento do consumidor (Morais \& Santos, 2015; Vargo \& Lusch, 2004; Gronröos, 2009), e o relacionamento entre consumidor e fornecedor pode influenciar a satisfação percebida, por meio da avaliação do nível de interações (Shankar, Smith, \& Rangaswamy, 2003). A cocriação é decisão do consumidor, e resulta de suas próprias escolhas para cocriar ou não com o fornecedor (Etgar, 2008), pois pode ser entendida como a sequência de atividades do cliente no contexto da criação de valor (Edvardsson, 1997; Eichentopf, Kleinaltenkamp, \& Van Stiphout, 2011). De maneira oposta, a coprodução sustenta-se no interesse organizacional, embora possa gerar valor resultante das experiências produtivas e de consumo do cliente (Morais \& Santos, 2015). 
Desse modo, se há percepção de valor pelo consumidor no conjunto de ações de cocriação e de coprodução de serviços, o resultado desta percepção sobre a oferta (custos) e a troca (benefícios) se relaciona ao bem e torna a satisfação uma consequência decorrente do valor percebido (Zeithaml, 1988; Zeithaml, Bitner, \& Gremler, 2006). A relação participativa na cocriação e na coprodução agrega valor (Morais \& Santos, 2015) e pode refletir em decisões futuras de relacionamento (Thompson \& Malaviya, 2013), de acordo com a satisfação do consumidor, (Zeithaml, Bitner, \& Gremler, 2006). O consumidor internaliza a experiência vivenciada com o prestador de serviços, que pode reforçar a confiabilidade e a integridade percebida em relação ao fornecedor (Singh \& Sirdeshmukh, 2000). Então,

H3: é o valor cocriado na experiência de cocriação e de coprodução que determina a satisfação percebida pelo consumidor.

Destaca-se, ainda, que os níveis diferentes de engajamento do consumidor (Morais \& Santos, 2015) pode alterar as bases de interação e, consequentemente, de satisfação (Prahalad \& Ramaswamy, 2004). Logo, a percepção de satisfação do consumidor pode ser influenciada pela forma de experiências, com a percepção diferentes em níveis de satisfação entre os serviços cocriados ou coproduzidos.

Identifica-se, na teoria, que se os processos de cocriação e de coprodução possuem diferenças em níveis de interação e de engajamento do consumidor (Morais \& Santos, 2015; Vargo \& Lusch, 2004; Gronröos, 2009) e a satisfação é uma consequência do valor percebido (Zeithaml, 1988; Zeithaml, Bitner, \& Gremler, 2006), acredita-se que a cocriação e a coprodução (Morais \& Santos, 2015) podem apresentar níveis de satisfação diferentes em termos de valor percebido (Zeithaml, Bitner, \& Gremler, 2006).

Portanto, no âmbito desta pesquisa é possível que o consumidor que cocria e o consumidor que coproduz tenham percepções diferentes do valor cocriado e, consequentemente, da satisfação percebida. Desta forma, a hipótese sobre a influência da cocriação e da coprodução na percepção de satisfação,

H4: a cocriação e a coprodução de serviços, quando analisadas na composição da satisfação do consumidor, possuem impactos diferentes devido as diferenças no nível de engajamento do consumidor. 


\section{Metodologia}

A população-alvo deste estudo foram os clientes de bancos e de cooperativas de crédito de um município brasileiro localizado na região norte do país. O contexto de serviços financeiros foi escolhido devido ao alto envolvimento do cliente nos processos de cocriação (cooperativas de crédito) e coprodução (bancos) de serviços (Auh et al., 2007; Duarte et al., 2016). O atual cenário do segmento financeiro possui as melhores condições para se verificar e avaliar as escolhas disponíveis do cliente, ao cocriar e coproduzir serviços e identificar o efeito sobre o valor cocriado percebido. Outro aspecto considerado na seleção desta população-alvo para a pesquisa são as condições de avaliação da satisfação do cliente pós a coprodução ou a cocriação do serviço. Como a literatura evidencia, a cocriação e a coprodução resultam em valor cocriado para o consumidor e, consequentemente, maior satisfação.

A composição da amostra foi medida a partir da população de habitantes de um município brasileiro, localizado na região norte do país, e se considerou o indicador de população total divulgado pelo IBGE no censo de 2010 e atualizado por meio de estimativas populacionais anuais, consultado no ano de 2017 (IBGE, 2017). A amostra incorporou somente os clientes pessoa física e isso foi determinado pelo tipo de relação que exige engajamento e controle no processo de cocriação e coprodução de serviço.

Os termos cocriação e coprodução foram explicados aos respondentes pelos dois aplicadores da escala Likert de 7 pontos, ambos especialistas em administração. Devido aos desafios de selecionar uma amostra de consumidores que cocriam em serviços financeiros (este era o maior desafio), a amostra é não-probabilística, por conveniência, composta por 196 indivíduos. O tamanho da amostra foi calculado com base em uma população finita, conforme fórmula a seguir, onde:

Figura 2 - Cálculo Amostral (Fórmula Utilizada)

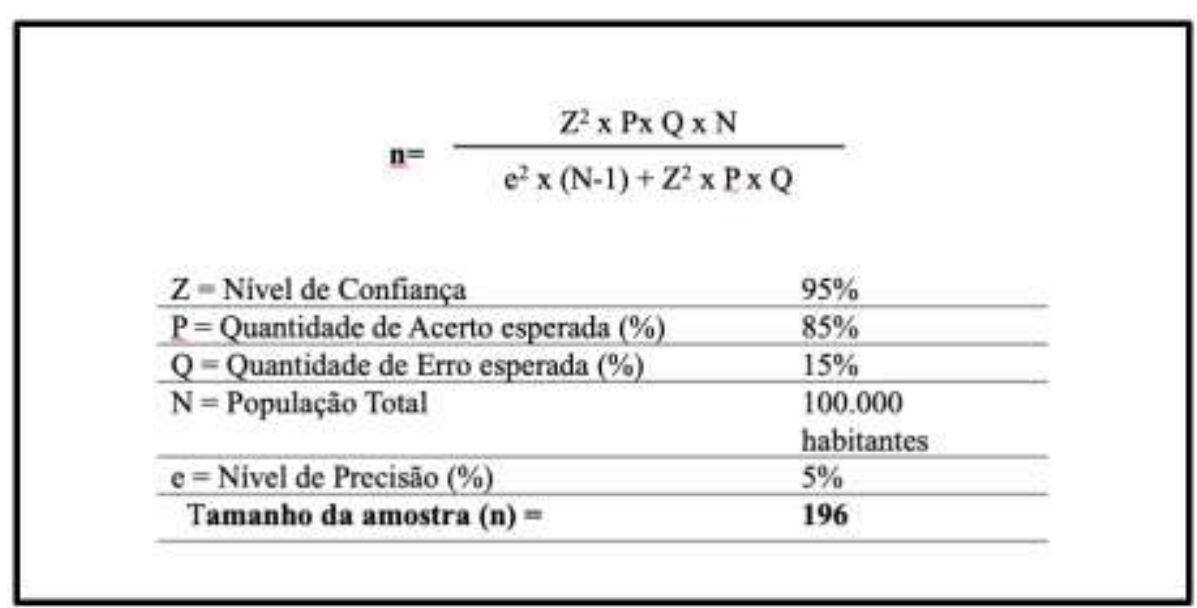

Fonte: Adaptado de McClave, Benson, \& Sincich, 2009. 
A delimitação geográfica teve como objetivo identificar uma população específica, pois a amostra global de clientes bancários tornaria inviável a pesquisa. Esta é uma das limitações do estudo e também uma oportunidade de pesquisa futura em outras regiões, como destacado nas considerações finais.

Para a construção da escala de medidas, foram utilizados componentes de escalas intervalares já validadas e apresentadas no Handbook of Marketing Scales (Bearden \& Netemeyer, 1999), sobre os seguintes temas: informação compartilhada, decisão compartilhada, aprendizagem conjunta, integração de recursos, flexibilidade de papeis, comunicação integrada e participação mútua (engajamento), para cocriação; e disponibilidade do cliente, disponibilidade de recursos, revisão de processos, normas explícitas, comunicação, educação do consumidor, interação, canais de comunicação, para coprodução (Morais \& Santos, 2015).

A escala utilizada foi do tipo Likert de 7 pontos, operacionalizada para medir a criação e a coprodução do cliente em sua percepção de valor cocriado e satisfação. Após a organização da escala foi feito um pré-teste com respostas de 66 pessoas, não incluídas na amostra principal.

A confiabilidade da escala foi verificada por meio da análise fatorial confirmatória, com uso do software SPSS 23.0 para verificação do ajuste do modelo, conforme apresentado na Tabela 1. Quanto às medidas de adequação da análise fatorial, o teste KMO (Kaiser-Meyer-Olkin) resultou em um valor de 0,614, considerado adequado. O teste Barlett de esfericidade mostrou-se significante (Qui-quadrado $=392,789253 ; \mathrm{df}=120 ; \mathrm{p}<0,001$ ), indicando que a análise fatorial foi adequada (Hair et al., 2009). O teste KMO apresentou valor de 0,614, estatisticamente significante $(p<0,01)$.

Portanto, reforça-se que o valor para o teste de esfericidade de Bartlett é satisfatório, isto é, $>0,05$, e o critério KMO tem parâmetros aceitáveis para as correlações $<0,5$, onde ressalta-se que os indicadores desta pesquisa apresentaram correlação superiores a 0,5 e dimensões com confiabilidade superior a 0,7, como evidenciado pela literatura (Hair et al., 2009). Evidencia-se, ainda, que a configuração dos fatores cocriação e coprodução estão dispostos conforme o agrupamento fatorial e, por último, cabe, ainda, destaca que o modelo segue sua versão primária, sem ajustes em suas variáveis ou atributos que o compõe, sem qualquer exclusão de itens, como demonstrado na tabela a seguir (TABELA 1). A seguir apresenta-se a tabela com as variáveis analisadas e suas respectivas cargas fatoriais, agrupadas nos construtos coprodução, cocriação, percepção de valor cocriado e satisfação percebida, como descrito na primeira coluna (constructos). 
Tabela 1 - Variáveis analisadas

\begin{tabular}{|c|c|c|c|c|}
\hline Constructo & Item & Correlação & $\boldsymbol{C F}$ & $\begin{array}{l}\text { a } \\
\text { Cronbach }\end{array}$ \\
\hline \multirow{8}{*}{ Coprodução } & Disponibilidade do cliente em coproduzir (COP_1) & 0,704 & 0,576 & \multirow{8}{*}{0,728} \\
\hline & Disponibilidade de recursos para coproduzir (COP_2) & 0,697 & 0,702 & \\
\hline & $\begin{array}{l}\text { Revisão de processos para se ajustar à coprodução } \\
\text { (COP_3) }\end{array}$ & 0,881 & 0,554 & \\
\hline & Explicitação de normas para a coprodução (COP_4) & 0,680 & 0,663 & \\
\hline & Fluidez na comunicação (COP_5) & 0,708 & 0,757 & \\
\hline & $\begin{array}{l}\text { Bases de educação do consumidor para a coprodução } \\
\text { (COP_6) }\end{array}$ & 0,773 & 0,811 & \\
\hline & Ambientes viáveis para a interação (COP_7) & 0,715 & 0,563 & \\
\hline & $\begin{array}{l}\text { Adequação dos canais de comunicação para a } \\
\text { coprodução (COP_8) }\end{array}$ & 0,714 & 0,706 & \\
\hline \multirow{8}{*}{ Cocriaçãa } & $\begin{array}{l}\text { Compartilhamento de informações na cocriação } \\
\text { (COC_1) }\end{array}$ & 0,670 & 0,801 & \multirow{8}{*}{0,707} \\
\hline & Decisão compartilhada sobre a cocriação (COC_2) & 0,642 & 0,719 & \\
\hline & Revisão de processos para a cocriação (COC_3) & 0,571 & 0,604 & \\
\hline & Aprendizagem conjunta com a cocriação (COC_4) & 0,690 & 0,664 & \\
\hline & $\begin{array}{l}\text { Integração de recursos entre as partes para a cocriação } \\
\text { (COC_5) }\end{array}$ & 0,528 & 0,754 & \\
\hline & Flexibilidade de papeis na cocriação (COC_6) & 0,624 & 0,841 & \\
\hline & Comunicação Integrada na cocriação (COC_7) & 0,625 & 0,522 & \\
\hline & Participação mútua na cocriação (COC_8) & 0,634 & 0,523 & \\
\hline \multirow{2}{*}{$\begin{array}{lll}\text { Percepção } & \text { de } & \text { Valor } \\
\text { Cocriado } & & \end{array}$} & Percepção de valor cocriado na cocriação (VCC) & 0,530 & 0,686 & \multirow{2}{*}{0,634} \\
\hline & Percepção de valor cocriado na coprodução (VCP) & 0,465 & 0,695 & \\
\hline \multirow{3}{*}{ Satisfação Percebida } & Satisfação percebida (COP) & 0,554 & 0,554 & \multirow{3}{*}{0,554} \\
\hline & Satisfação percebida (COC) & 0,554 & 0,554 & \\
\hline & Satisfação percebida (VC) & 0,554 & 0,554 & \\
\hline
\end{tabular}

Fonte: Morais \& Santos (2015, p. 245) - Escala adaptada de Handbook of Marketing Scales (Bearden \& Netemeyer, 1999).

\section{Análise dos dados}

De acordo com Arbuckle (2006) e Anderson e Gerbing (1988), utilizaram-se duas etapas para realizar o procedimento de análise da SEM com o AMOS 24.0. A primeira etapa foi composta pelos critérios de qualidade por meio da Análise Fatorial Confirmatória (AFC), para assegurar a confiabilidade e a validade dos dados, ao testar as relações causais entre as variáveis latentes, na segunda etapa. A validade convergente apresentou variância média extraída com valor mínimo acima de 0,4 e as cargas fatoriais acima de 0,5. Embora não sejam valores representativos, mas são aceitáveis para as condições do modelo. A validade discriminante entre as variáveis latentes foi confirmada, já que a correlação não excedeu a variância extraída ao quadrado (variáveis latentes) e 
as cargas fatoriais dos itens foram maiores em seus respectivos construtos do que em outras variáveis (TABELA 2).

Tabela 2 - Validade discriminante das variáveis latentes

\begin{tabular}{|l|c|c|c|c|}
\hline & Cocriação & Coprodução & Valor Cocriado Percebido & Percepção de Satisfação \\
\hline Cocriação & 0,52 & & & \\
\hline Coprodução & - & 0,50 & & \\
\hline Valor Cocriado Percebido & - & - & 0,58 & 0,54 \\
\hline Percepção de Satisfação & - & - & - & \\
\hline
\end{tabular}

Fonte: Dados da pesquisa.

A avaliação do ajuste (goodness-of-fit - GOF) foi feita por múltiplos indicadores: $\chi 2$ (quiquadrado), $\chi 2$ / df (qui-quadrado para graus de liberdade), CFI (índice de ajuste comparativo), GFI (índice de bom ajuste do modelo), TLI (índice de Tucker Lewis), e RMSEA (erro quadrático médio de aproximação). Segundo Hair et al. (2009), o ajuste do modelo é bom quando os índices $\geq 0.90, \chi$ 2/df entre 2 e 5 e RMSEAs $\leq 0.08$.

Figura 3 - Modelo fit

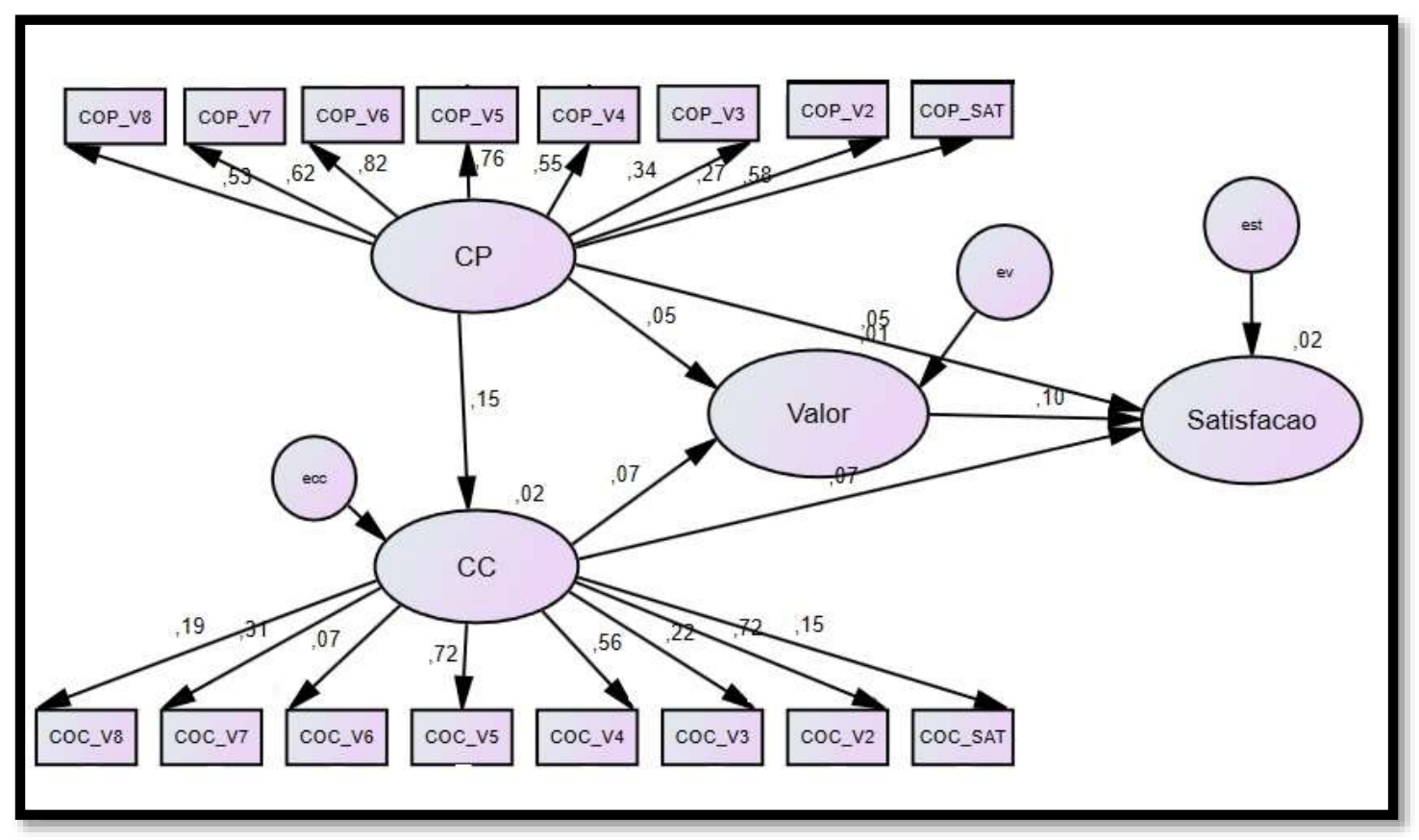

Fonte: Dados da pesquisa.

Este modelo apresentou $\chi 2(211,642), \chi 2$ / df $(211,642$ / $103=2,055)$, CFI $(0,625)$, TLI $(0,505)$ e RMSEA $(0,153)$, e pode-se afirmar que todas as estatísticas estão próximas dos valores aceitáveis (Hair et al., 2009). No entanto, não é suficiente para afirmar que há efeito da coprodução e da cocriação sobre o valor cocriado e a satisfação do cliente. A correlação de Pearson não foi significante $(\mathrm{p}<0,01)$ entre as variáveis coprodução e cocriação e valor cocriado e valor coproduzido. Conforme o modelo gráfico, a única afirmação possível é sobre a existência de 
correlação entre as variáveis de coprodução e de cocriação, que possuem capacidade explicativa significantes para a ação conjunto produtiva ou de criação (Morais \& Santos, 2015). O baixo poder de explicação da coprodução e da cocriação sobre o valor cocriado ou coproduzido e a satisfação do cliente pode representar necessidade de ajuste do modelo ou revisão dos constructos de valor cocriado.

As estimativas dos coeficientes padronizados destacam que a relação entre a coprodução e o valor coproduzido $(\beta=0,05 ; p<0,01)$, e entre a coprodução e a satisfação $(\beta=0,05 ; \mathrm{p}<0,01)$ mostram baixa capacidade de explicação. De maneira similar, as estimativas dos coeficientes padronizados destacam que entre a cocriação e o valor cocriado $(\beta=0,07 ; p<0,01)$, e entre a cocriação e a satisfação $(\beta=0,07 ; p<0,01)$ também há baixa capacidade de explicação. Consequentemente, as estimativas dos coeficientes padronizados mostram que o caminho entre o valor cocriação e a satisfação $(\beta=0,10 ; p<0,01)$ também não é representativo. Assim, os resultados mostraram que o modelo estrutural não alcançou um nível de ajuste satisfatório, no qual $\chi 2$ $(211,642), \chi 2$ / df $(211,642$ / $103=2,055)$, CFI $(0,625)$, TLI $(0,505)$ e RMSEA $(0,153)$, apresentam estatísticas próximas dos valores aceitáveis (Hair et al., 2009). Os resultados indicam que a única relação sustentada é entre coprodução e cocriação $(\beta=173, \mathrm{p}=0,01)$, dando apoio à teoria LDS, que destaca que serviços são sempre coproduzidos ou cocriados.

Diante dos dados expostos, cabe destacar que o modelo não se apresentou satisfatório quanto aos elementos teóricos. Há que se ponderar sobre as limitações do modelo SEM em análise, pois existem elementos da própria pesquisa que podem ter contribuído para os resultados encontrados. A amostra em um único município brasileiro, de região periférica do país, e população amostral delimitada pela acessibilidade aos respondentes, podem representar um limitador que, se replicada com seleção amostral com técnicas aleatórias e maior diversidade regional (representatividade nacional), podem gerar resultados diferentes daquele identificados.

Outro aspecto importante e que pode contribuir para as limitações é o contexto de serviço em análise (bancos e cooperativas de crédito). A teoria destaca que serviços são sempre cocriados e/ou coproduzidos, mas a intensidade (nível) de serviço e a tipo de necessidade do consumidor podem alterar o engajamento e, consequentemente, alterar a percepção de valor e de satisfação decorrente da cocriação e a da coprodução. Outros setores de serviços, como exemplo de saúde e de educação, podem apresentar resposta diferentes e com maior impacto devido ao maior envolvimento no processo de cocriação e de coprodução.

Embora a teoria analisada enfatiza o modelo teórico em foco e haja resultados similares ou complementares a estes, em outros estudos, o nível de aceitabilidade dos indicadores estatísticos impõem dúvida quanto a construção do modelo ideal. Neste ponto, cabe mencionar a contribuição de um dos avaliadores que destacou as diferenças teóricas evidenciadas neste estudo sobre os 
clientes associados às cooperativas de créditos, pois discute-se na teoria a existência de características que os diferenciam dos clientes bancários. Esta constatação remeterá implicações para a SEM, pois, se há distinção nas características do público, teria maior contribuição e rigor se fosse analisada a cocriação e coprodução separadamente e realizada comparação.

\section{Discussão dos resultados}

Para a análise dos resultados apresentados anteriormente é necessário retomar alguns elementos já discutidos no recorte teóricos utilizado por este estudo. Isso se faz necessário ao explicar e sustentar a contribuição deste estudo em relação ao arcabouço teórico existente. Identifica-se que há aproximadamente quinze anos a teoria de gestão vem construindo e discutindo modelos de referência para a cocriação de valor, cujo objetivo é representar a construção do valor conjunto e medir o valor cocriado nas relações de negócios. Como exemplos já mencionados anteriormente, o modelo proposto por Prahalad e Ramaswamy (2004) foca no ambiente que estimula a interação entre o consumidor e o fornecedor, por meio do diálogo, do acesso, do confronto entre riscos e benefícios e da transparência nas relações entre os players. O Modelo DART (Diálogo, Acesso, Risco/Benefício e Transparência) pressupõe a combinação dos quatro pilares para o engajamento dos consumidores nos processos da empresa, pois, do contrário, se não forem utilizados em conjunto, tornam-se apenas facilitadores no processo de coprodução/cocriação, sem garantias de valor cocriado.

De forma complementar ao modelo de Prahalad e Ramaswamy (2004), o modelo de cocriação de experiência em relacionamento com a marca, proposto por Payne et al. (2008), pressupõe a compreensão do processo de valor (que/como?) para entender o que é o valor cocriado e o que facilita a sua construção. Essa concepção de modelo de cocriação de valor é baseada em um conjunto de ferramentas utilizadas em processos, atividades e tarefas, por meio de procedimentos, mecanismos e interações, que se sustentados em proposições de valor, tornam-se capazes de gerar resultados de valor cocriados. Logo, o cliente é o cocriador de valor, e o valor é fato que só ocorre no uso ou na experiência vivenciada e percebida.

Por último, o modelo de valor em uso, proposto por Grönroos (2011), assemelha-se aos modelos anteriores, pois acredita-se que a cocriação de valor é decorrente do processo conjunto no desenvolvimento de serviço/produto, mas difere dos modelos apresentados anteriormente quanto ao processo de cocriação do valor. O autor defende que o desenvolvimento de serviço/produto é realizado conscientemente pelo consumidor em seu envolvimento na produção/criação, mas o valor cocriado, de forma geral, é percebido de modo inconsciente pelo consumidor, e emerge quando o fornecimento do serviço é realizado ou no uso do produto. Desse modo, a cocriação de valor é dependente da percepção de valor que emerge do uso, ou em condições específicas quando o 
consumidor é envolvido na produção/criação (design, desenvolvimento, confecção, parte do processo). Portanto, é importante que as empresas absorvam as necessidades e as expectativas dos consumidores, a fim de interagir e influenciá-los na cocriação de valor, pois acreditar que o consumidor é sempre um cocriador de valor, pode ser um equívoco sobre seu papel na cocriação do valor.

Entretanto, cabe destacar que estes modelos anteriores tiveram como objetivos apresentar evidências teóricas sobre o que é a cocriação de valor e como ela ocorre no engajamento dos consumidores (Prahalad \& Ramaswamy, 2004), na produção conjunta entre empresa e consumidores, na experiência vivenciada e percebida pelo consumidor (Payne et al., 2008) e na especificidade do contexto de uso dos consumidores (Grönroos, 2011). O modelo estudado neste artigo foi elaborado com o objetivo de propor um modelo teórico de mensuração da cocriação de valor (Morais \& Santos, 2015). Assim, o primeiro ponto a ser discutido acerca das contribuições deste estudo é sobre os limites conceituais que compõem e sustentam os elementos que formam o constructo coprodução ou o constructo cocriação.

A dimensão coprodução (constructo 1) é composta por oito categorias (variáveis observadas), a saber: disponibilidade do cliente, disponibilidade de recursos, revisão de processos, normas explícitas, fluidez na comunicação, educação do consumidor, ambiente de interação, canais de contato. Por sua vez, a dimensão de cocriação (constructo 2) é composta por sete categorias (variáveis observadas): informação e decisão compartilhadas, revisão de processo, aprendizagens conjuntas, integração de recursos, flexibilidade de papéis, comunicação integrada, engajamento mútuos.

Neste contexto, há evidências estatística suficientes para se afirmar que a cocriação e a coprodução são constructos que se compõem por meio das variáveis mencionadas anteriormente, conforme agrupamento fatorial exposto nos dados ( $\alpha$ Cronbach). Além disso, pode-se afirmar que a ação de coprodução com fornecedores pode evoluir para a cocriação em serviços (hipótese H1), de acordo com a percepção dos consumidores $\left(\mathrm{R}^{2} 0,15\right)$. Identifica-se que a coprodução, como a teoria abordada destaca, pode exigir da coprodução o incremento de ações da cocriação, por meio de mudança no papel do consumidor dentro do processo de coprodução. Isto é, altera-se o papel de consumidor coprodutor e o estende a participação do consumidor na concepção de novos processos, novas melhorias e utilidade do produto/serviço ou de mercados.

Estes achados fazem com que este estudo promova contribuição teórica específica na organização de variáveis mencionadas no arcabouço teórico existente, conforme teste de significância para a correlação entre elas. Pode-se afirmar que as variáveis elencadas para a composição da coprodução e da cocriação estão correlacionadas e o formam os constructos de análise citados. Além disso, identifica-se que a coprodução pode evoluir para relacionamentos mais 
consistentes, quando o consumidor coprodutor passa a incorporar o papel de cocriador em um único processo de cocriação de valor para as partes interessadas.

Outro aspecto observado por este estudo é que, embora os efeitos para o valor cocriado e a satisfação do consumidor sejam estatisticamente significantes no modelo deste estudo $(p<0,05)$, ele possui baixa capacidade explicativa decorrentes da cocriação $\left(R^{2} 0,05\right)$ e da coprodução $\left(R^{2}\right.$ 0,07), fato que pode ser decorrente do tipo de engajamento e da percepção de valor do consumidor pesquisado (Grönroos, 2011). Desta proposição teórica emergem às hipóteses " $H 2 a$ " e "H2b" que o estudo confirma que há diferenças na capacidade de explicação do valor cocriado com a coprodução $(0,05)$ e valor cocriado com a cocriação $(0,07)$, embora não sejam diferenças expressivas (apenas $2 \%$ de diferença na explicação dos resultados).

A baixa diferença e representatividade do valor cocriado com a coprodução e a cocriação pode ter relação com o contexto em análise, cujo ambiente parece ser de baixo engajamento de acordo com a amostra pesquisada. Este é um outro ponto a ser discutido nas sugestões de pesquisa futura, pois, se a percepção de valor cocriado na coprodução de serviços é dependente do contexto produtivo e do engajamento dos consumidores, deveria haver maior distanciamento da capacidade explicativa do valor cocriado em processos de cocriação. Outros contextos de serviços, como exemplo, educação e saúde, podem exigir maior engajamento do consumidor. Portanto, de acordo com a percepção do consumidor participante do processo de cocriação e coprodução, embora estatisticamente ( $p$-value <0,05) exista diferenças em percepção de valor cocriado, no contexto analisado por este estudo, os resultados não são suficientes para se afirmar que há distinção em resultados de valor decorrente da coprodução e cocriação.

Por fim, destaca-se que o valor cocriado pode mediar a relação existente entre a cocriação e a coprodução, ainda que neste estudo se tenha uma explicação de média significância estatística. Destaca-se que a satisfação do consumidor pode ser explicada pela cocriação de valor $\left(R^{2} 0,10\right)$, mas o percentual de explicação parece não ser significativo para explicar a satisfação do consumidor, embora esteja próximo de médio para o campo de ciências sociais (Ringle, Silva, \& Bido, 2015). No entanto, o que antecipadamente parece uma limitação do estudo, como se mencionará a seguir, permite inferir análises sobre as tipologias de respondentes e de contexto de negócios. Nota-se que o nível e/ou intensidade de serviços altera o engajamento e a percepção de valor, como no caso de bancos e cooperativas de créditos, onde os serviços possuem substitutos em marcas e modelos de negócios e, provavelmente, há menor valor no uso ou na experiência vivenciada e percebida. Portanto, quanto mais essencial é o serviço (raro ou insubstituível) maior será a sua capacidade de explicar o valor cocriado (Payne et al. (2008).

Assim, este modelo contribui para ampliar a compreensão sobre o modo como ocorre a cocriação de valor na coprodução e na cocriação de serviços, especialmente ao identificar que a 
coprodução e a cocriação dependem de adicionais na esfera do consumidor, como exemplo da capacidade de a empresa engajá-los em ofertas únicas ou insubstituíveis e, assim, ter a capacidade de ampliar o valor percebido nos consumidores. Ademais, testar o arcabouço teórico existente em análise teórico-empírica (Morais \& Santos, 2015), estende-se o diálogo sobre a coprodução e a cocriação com fins na cocriação de valor, como discute-se a seguir.

\section{Considerações finais}

O principal objetivo da pesquisa foi identificar os efeitos da cocriação e da coprodução no valor cocriado e na satisfação do cliente. Os resultados mostraram que não há relação significativa entre a coprodução e a cocriação, e o valor cocriado e a satisfação do cliente. Esses resultados sugerem que os argumentos teóricos e práticos da coprodução e da cocriação com consumidores precisam ser revistos quanto aos objetivos (Gronröos, 2009). As possíveis explicações para estes resultados são: (1) viés amostral, dada a característica regional da amostra; (2) a coprodução é condição do fornecedor e o cliente pessoa física é instrumento produtivo e meio de redução de custos para o fornecedor; portanto, pode não perceber valor; (3) a gestão da cocriação com o cliente pode ser incipiente no mercado B2C, especialmente nas cooperativas de crédito pesquisadas, por isso não há valor percebido; e, (4) se a satisfação é decorrente do valor, não se pode falar em satisfação onde não há valor percebido.

Assim, embora existam estes elementos que lidam com o próprio conjunto de dúvidas desta pesquisa, pode-se afirmar que o artefato conceitual na literatura de gestão também é incerto no que se refere à coprodução e à cocriação para a construção de valor cocriado. São dilemas decorrentes de um framework parcial ou unidimensional, logo, sob a perspectiva teórica, este estudo apresenta como contribuição o desenvolvimento de medidas necessárias para obter informações sobre o fato dos serviços serem sempre cocriados ou coproduzidos, (i) mas pode-se afirmar que a cocriação e a coprodução, por si, não gera valor cocriado; (ii) outra contribuição deste estudo é identificar que diferentes modelos utilizados para a medição do valor cocriado evidenciam a dependência de perspectiva conceitual de valor para os resultados de efeito, pois existem diferenças se comparado a outros modelos propostos pela literatura de gestão acerca do valor cocriado e dos elementos conceituais de construção do valor com a coprodução e a cocriação; e, por último, destaca-se que a variável dependente satisfação do consumidor recebe pouca explicação estatística da coprodução $\left(\mathrm{R}^{2} 0,7\right)$ e da cocriação $\left(\mathrm{R}^{2} 0,5\right)$, quando isoladas, portanto a pouca capacidade de explicação destas variáveis ressaltam que a cocriação e a coprodução, por si, não gera satisfação ao cliente. De outra forma, quanto ao valor cocriado $\left(\mathrm{R}^{2} 0,10\right)$, há limitações para se fazer inferências sobre a capacidade de explicação para a satisfação, pois as estatísticas no valor cocriado possuem baixa significância enquanto resultado da coprodução $\left(R^{2} 0,05\right)$ e da cocriação $\left(R^{2} 0,07\right)$, portanto, as diferenças 
observadas entre a teoria e os resultados podem ser decorrentes do próprio recorte teórico do modelo e não da capacidade do valor cocriado gerar ou explicar a satisfação do consumidor.

As contribuições gerenciais deste estudo apontam para a maior atenção dos gestores à gestão da coprodução e da cocriação. A teoria destaca que o valor cocriado na cocriação e na coprodução é decorrente de determinantes da interação e do engajamento com o cliente. No entanto, apesar de haver significância para a cocriação e a coprodução, estas ações não têm significância para o valor percebido ou a satisfação. Isto pode indicar, no caso da amostra desta pesquisa, que os clientes de serviços financeiros coproduzem ou cocriam na extensão do fornecedor e não do cliente. Como o valor é contextual e dependente de contingências dos atores, dos recursos e do relacionamento em situações específicas e únicas (Prahalad \& Ramaswamy, 2004; Edvardson, Tronvoll, \& Gruber, 2011), ressalta-se que o contexto desta pesquisa necessita de maior investigação teórica e atenção gerencial, para identificar as lacunas destacadas no modelo quanto à ausência de significância para o valor cocriado e a satisfação do cliente.

Observa-se que a teoria ainda não está consolidada e os modelos construídos apresentam limitações, principalmente quanto à completude dos constructos e repostas a contextos e macroambientes (Leroy, Cova, \& Salle, 2012; Morais \& Santos, 2015). Assim, como proposta de agendas futuras de pesquisa, sugere-se testar novas categorias teóricas e novas modelagens, que poderão contribuir para a organização e a ampliação das categorias propostas neste modelo, e a produção de outras métricas para a coleta de dados e testes empíricos. Identificou-se, também, informação teóricas que indicam que clientes associados às cooperativas de créditos possuem características diferentes de clientes bancários e, se analisado e comparado separadamente, caso seja proposição verdadeira, a indicação das características do público das cooperativas de créditos na cocriação de valor distaria do público de bancos tradicionais e essa verificação poderia trazer novas análises em pesquisa futuras. Por último, reforça-se a proposta de Morais e Santos (2015) sobre pesquisas que refinem as ideias de coprodução, cocriação, cocriação de valor e valor percebido, em outras bases teóricas além da LDS.

\section{Referências}

Alford, J. (2002). Por que os clientes do setor público coproduzam? Rumo a uma teoria da contingência. Administração \& Sociedade , 34 (1), 32-56.

Agrawal, A. K., \& Rahman, Z. (2015). Roles and resource contributions of customers in value cocreation. International Strategic Management Review, 3(1-2), 144-160.

Anderson, J. C., \& Gerbing, D. W. (1988). Structural equation modeling in practice: A review and recommended two-step approach. Psychological bulletin, 103(3), 411. 
Arasli, H., Mehtap-Smadi, S., \& Turan Katircioglu, S. (2005). Customer service quality in the Greek Cypriot banking industry. Managing Service Quality: An International Journal, 15(1), 41-56.

Arbuckle, J. L. (2006). Amos (version 7.0)[computer program]. Chicago: SpSS.

Auh, S., Bell, S. J., McLeod, C. S., \& Shih, E. (2007). Co-production and customer loyalty in financial services. Journal of retailing, 83(3), 359-370.

Bearden, W. O., \& Netemeyer, R. G. (1999). Handbook of marketing scales: Multi-item measures for marketing and consumer behavior research. Sage.

Bendapudi, N., \& Leone, R. P. (2003). Psychological implications of customer participation in coproduction. Journal of marketing, 67(1), 14-28.

de Souza Bido, D., de Souza, C. A., da Silva, D., Schmidt Godoy, A., \& Rivera Torres, R. (2012). Qualidade do Relato dos Procedimentos Metodológicos em Periódicos Nacionais na Área de Administração de Empresas: o caso da modelagem em equações estruturais nos periódicos nacionais entre 2001 e 2010. Organizações \& Sociedade, 19(60).

Chandler, J. D., \& Vargo, S. L. (2011). Contextualization and value-in-context: How context frames exchange. Marketing theory, 11(1), 35-49.

Chathoth, P., Altinay, L., Harrington, R. J., Okumus, F., \& Chan, E. S. (2013). Co-production versus co-creation: A process based continuum in the hotel service context. International Journal of Hospitality Management, 32, 11-20.

Duarte, A. F., Moreira, V. R., Ferraresi, A. A., \& Gerhard, A. (2016). Evaluating credit union members' perception of service quality through service innovation. RAI Revista de Administração e Inovação, 13(4), 242-250.

Edvardsson, B. (1997). Quality in new service development: Key concepts and a frame of reference. International Journal of Production Economics, 52(1-2), 31-46.

Eichentopf, T., Kleinaltenkamp, M., \& Van Stiphout, J. (2011). Modelling customer process activities in interactive value creation. Journal of Service Management, 22(5), 650-663.

Etgar, M. (2008). A descriptive model of the consumer co-production process. Journal of the academy of marketing science, 36(1), 97-108.

Galvagno, M., \& Dalli, D. (2014). Theory of value co-creation: a systematic literature review. Managing Service Quality, 24(6), 643-683.

Grönroos, C. (2009). Towards service logic: The unique contribution of value co-creation.

Grönroos, C. (2011). A service perspective on business relationships: The value creation, interaction and marketing interface. Industrial marketing management, 40(2), 240-247.

Grönroos, C., \& Voima, P. (2013). Critical service logic: making sense of value creation and cocreation. Journal of the academy of marketing science, 41(2), 133-150. 
Hair, J. F., Black, W. C., Babin, B. J., Anderson, R. E., \& Tatham, R. L. (2009). Análise multivariada de dados. Bookman Editora.

Lusch, R. F., \& Vargo, S. L. (2006). Service-dominant logic: reactions, reflections and refinements. Marketing theory, 6(3), 281-288.

Kauffmann, S., \& Marchetti, R. Z. (2008). Canais de atendimento bancário e satisfação do cliente: um estudo em bancos de varejo. Encontro De Marketing Da Associação Nacional Dos Programas De Pós-Graduação Em Administração, 3.

McClave, J. T., Benson, P. G., \& Sincich, T. Estatística para Administração e Economia. 10. Ed. São Paulo: Pearson Prentice Hall, 2009

Morais, F. R., \& Santos, J. B. (2015). Refinando Os Conceitos De Cocriação E Coprodução: Resultados De Uma Crítica Da Literatura. Revista Economia \& Gestão, 15(40), 224-250.

Nambisan, S., \& Baron, R. A. (2007). Interactions in virtual customer environments: Implications for product support and customer relationship management. Journal of interactive marketing, 21(2), 42-62.

Nambisan, S., \& Baron, R. A. (2009). Virtual customer environments: testing a model of voluntary participation in value co- creation activities. Journal of product innovation management, 26(4), 388-406.

Ojasalo, K. (2010). The shift from co-production in services to value co-creation. The Business Review, Cambridge, 16(1), 171-177.

Ostrom, E. (1996). Crossing the great divide: coproduction, synergy, and development. World development, 24(6), 1073-1087.

Parasuraman, A., Zeithaml, VA e Berry, LL (1985). Um modelo conceitual de qualidade do serviço e suas implicações para futuras pesquisas. o Journal of Marketing , 41-50.

Payne, A. F., Storbacka, K., \& Frow, P. (2008). Managing the co-creation of value. Journal of the academy of marketing science, 36(1), 83-96.

Pohlmann, A., \& Kaartemo, V. (2017). Research trajectories of Service-Dominant Logic: Emergent themes of a unifying paradigm in business and management. Industrial Marketing Management, 63, 53-68.

Prahalad, C. K., \& Ramaswamy, V. (2000). Co-opting customer competence. Harvard business review, 78(1), 79-90.

Prahalad, C. K., \& Ramaswamy, V. (2004). Co- creation experiences: The next practice in value creation. Journal of interactive marketing, 18(3), 5-14.

Prandelli, E., Verona, G., \& Raccagni, D. (2006). Diffusion of web-based product innovation. Califoronia Management Review, 48(4), pp. 109-135.

Ramirez, R. (1999). Value co-production: intellectual origins and implications for practice and research. Strategic management journal, 49-65. 
Ribeiro, J. L. D., Machado, C. O., \& Tinoco, M. A. C. (2010). Determinantes da satisfação e atributos da qualidade em serviços bancários. Gestão e produção. São Carlos, SP. Vol. 17, n. 4 (out./dez. 2010), p. 775-790.

Shankar, V., Smith, A. K., \& Rangaswamy, A. (2003). Customer satisfaction and loyalty in online and offline environments. International journal of research in marketing, 20(2), 153-175.

Singh, J., \& Sirdeshmukh, D. (2000). Agency and trust mechanisms in consumer satisfaction and loyalty judgments. Journal of the Academy of marketing Science, 28(1), 150-167.

Thompson, D. V., \& Malaviya, P. (2013). Consume-generated ads: does awareness of advertising co-creation heip or hurt persuasion. Journal of Marketing, 77 , pp. 33-47.

Vargo, S. L., \& Lusch, R. F. (2004). Evolving to a new dominant logic for marketing. Journal of marketing, 68(1), 1-17.

Williams, P., \& Naumann, E. (2011). Customer satisfaction and business performance: a firmlevel analysis. Journal of services marketing, 25(1), 20-32.

Yin, Chan, \& Lam. (Novembro de 2012). Do Customers and Employees Enjoy Service Participation? Synergistic Effects of Seif- and Other-Efficacy. Journal of Marketing, 76, 121140.

Zeithaml, V., Bitner, M. J., \& Gremler, D. (2006). Services marketing: Integrating customer focus across the firm. McGraw Hill.

Zeithaml, V. A. (1988). Consumer perceptions of price, quality, and value: a means-end model and synthesis of evidence. The Journal of marketing, 2-22.

Wu, L. W., Wang, C. Y., \& Rouyer, E. (2019). The opportunity and challenge of trust and decision-making uncertainty. International Journal of Bank Marketing. 
ANEXO I - Instrumento de coleta de dados

\section{INDENTIFICAÇÃO:}

b) Sexo: ( ) M ( ) F

c) Nível de escolaridade?

( ) nível fundamental incompleto ( ) nível fundamental completo ( ) nível médio incompleto

$\overline{\text { ( ) nível médio completo ( ) nível superior incompleto ( ) nível superior }}$
completo

d) Há quanto tempo é cooperado de uma cooperativa de crédito ou é cliente do Banco, conforme seu caso específico? Sou cliente/Cooperado no Banco/Cooperativa há anos.

\section{Coprodução de Serviços Bancários}

Assinale o número que melhor representa a sua relação com a cooperativa de crédito ou o banco, conforme o seu caso (1 para NÃO CONCORDO e 7 para CONCORDO TOTALMENTE):

1. Eu estou sempre disponível para realizar os meus próprios serviços bancários, isto é, importa para mim produzir nos autos serviços do meu banco e atender as minhas próprias necessidades de modo autossuficiente e independente.

\begin{tabular}{lllllll}
\hline 1 & 2 & 3 & 4 & 5 & 6 & 7 \\
\hline 2. Eu sempre tenho disponibilidade de recursos para coproduzir com o meu banco,
\end{tabular} 2. Eu sempre tenho disponibilidade de recursos para coproduzir com o meu banco,
como exemplo de recursos eletrônicos compatíveis com o banco (computadores, tabletes e smartphones).

\begin{tabular}{lllllll}
\hline 1 & 2 & 3 & 4 & 5 & 6 & 7 \\
\hline
\end{tabular}

3. Sempre que eu preciso o meu banco revisa ou muda os processos para se ajustar as minhas necessidades de autosserviços e de produção para se adequar as minhas necessidades.

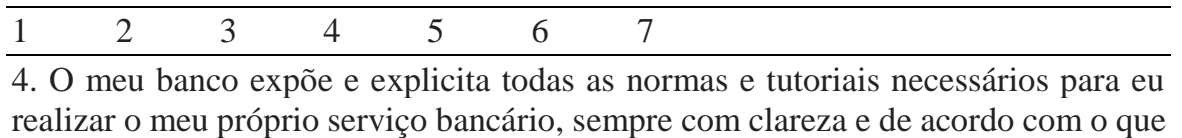
o é melhor para mim. $\begin{array}{lllllll}1 & 2 & 3 & 4 & 5 & 6 & 7\end{array}$

5. No meu banco a comunicação é fluida e existem vários canais de contato direto com o cliente. Estou em contato constante e direto, pois não há barreiras na comunicação entre eu e o banco.

$\begin{array}{lllllll}1 & 2 & 3 & 4 & 5 & 6 & 7\end{array}$

6. O meu banco provê os recursos necessários para que eu consiga realizar os meus serviços e tenha uma educação bancaria sólida enquanto consumidor e produtor do meu próprio serviço.

\begin{tabular}{lllllll}
1 & 2 & 3 & 4 & 5 & 6 & 7 \\
\hline 7. & No meu banco existem ambientes viáveis para a interação, com flexibilidade de
\end{tabular} comunicação, execução de serviços, com a possibilidade de eu realizar diferentes ações na autoprodução dos meus serviços bancários.

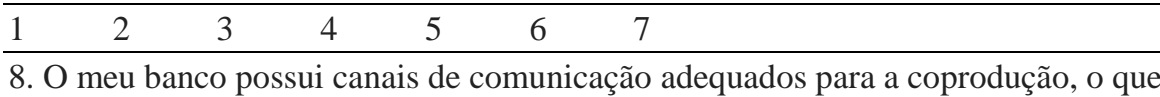
facilita o uso dos recursos disponíveis para realizar meus próprios serviços.

\begin{tabular}{lllllll}
\hline 1 & 2 & 3 & 4 & 5 & 6 & 7
\end{tabular}

\section{Cocriação - Produto/Serviço em Cooperativa de Crédito}

1. As informações são compartilhadas pela cooperativa de crédito e são altamente confiáveis.

$\begin{array}{lllllll}1 & 2 & 3 & 4 & 5 & 6 & 7\end{array}$

2. Eu procuro participar de todas as atividades da cooperativa de crédito, incluindo assembleias e decisões. Eu vejo a cooperativa de crédito como minha casa/empresa e não como banco.

$\begin{array}{lllllll}1 & 2 & 3 & 4 & 5 & 6 & 7\end{array}$

3. Eu uso diversos serviços da cooperativa de crédito, pois ajudei planejá-los para se adequar as minhas necessidades, já que os bancos não fariam o mesmo.

\begin{tabular}{lllllll}
\hline 1 & 2 & 3 & 4 & 5 & 6 & 7
\end{tabular}

4. Sempre que participo dos serviços ofertados pela cooperativa de crédito há novas aprendizagens, pois eles me escutam quando falo de minhas necessidades e eles me explicam como é melhor para mim. Assim aprendemos juntos.

\begin{tabular}{lllllll}
\hline 1 & 2 & 3 & 4 & 5 & 6 & 7 \\
\hline 5 & Eu coloco & recursos na cooperativa de crédito e também uso os recursos
\end{tabular} disponíveis. Isso ocorre porque os recursos integrados são nossos. $\begin{array}{lllll}2 & 3 & 4 & 5 & 6\end{array}$

Braz. Jour. Mark. - BJM

Rev. Bras. Mark - ReMark, São Paulo, 19(1), p. 117-140, Jan./Mar. 2020 
6. Na cooperativa de crédito existem momentos em que sou cliente, mas também sou dono. Há flexibilidade de papéis e exige ações diferentes do cooperado.

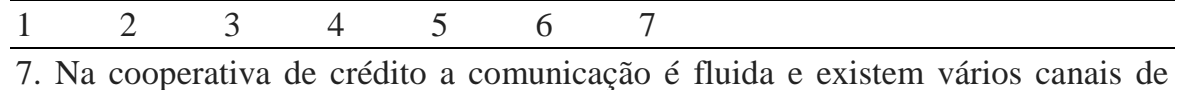
contato direto com o cooperado. Estamos em contato constante e não há barreiras na comunicação.

\begin{tabular}{lllllll}
\hline 1 & 2 & 3 & 4 & 5 & 6 & 7 \\
\hline
\end{tabular}

8. Todos somos um. Na cooperativa de crédito o nosso maior bem é o engajamento mutuo, não existindo o eu. Os resultados alcançados são para nós.

\begin{tabular}{lllllll}
\hline 1 & 2 & 3 & 4 & 5 & 6 & 7 \\
\hline
\end{tabular}

\section{Percepção de Valor Cocriado}

9. Sobre os serviços prestados pelo meu banco e coproduzidos por mim, eu percebo que isso gera valor conjunto decorrente do tipo de relação. $\mathrm{O}$ meu banco gera valor mútuo quando eu coprodução os meus serviços.

\begin{tabular}{lllllll}
\hline 1 & 2 & 3 & 4 & 5 & 6 & 7 \\
\hline 9. & Na minha cooperativa de crédito as decisões são tomadas de maneira clara e
\end{tabular} participativa, de modo que seja bom para o cooperado e tenha garantias de participação na criação de serviços e produtos da cooperativa de crédito, fato que gera valor conjunto decorrente deste tipo de relação, ao criar produtos serviços conjuntamente.

\begin{tabular}{lllllll}
\hline 1 & 2 & 3 & 4 & 5 & 6 & 7 \\
\hline
\end{tabular}

\section{Percepção de Satisfação}

10. Sobre o seu relacionamento com a cooperativa de crédito, assinale a nota que melhor representa a sua satisfação.

\begin{tabular}{|c|c|c|c|c|c|c|}
\hline 1 & 2 & 3 & 4 & 5 & 6 & 7 \\
\hline \multicolumn{7}{|c|}{$\begin{array}{l}\text { 11. Sobre o seu relacionamento com o banco, assinale a nota que melhor representa } \\
\text { a sua satisfação. }\end{array}$} \\
\hline 1 & 2 & 3 & 4 & 5 & 6 & 7 \\
\hline
\end{tabular}
cooperativa, de a nota que melhor representa a sua satisfação. 



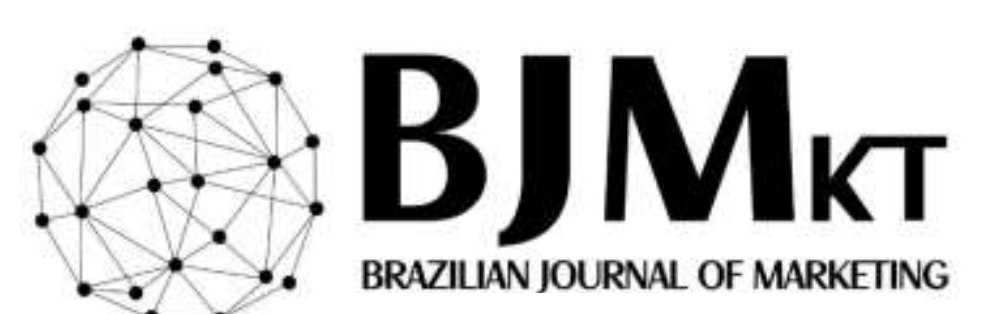

Received: 28 Mar. 2018 / Approved: 29 Jan. 2020 Responsible editors: Julio Araujo Carneiro da Cunha \& André Torres Urdan

Evaluation: Double Blind Review e-ISSN: $2177-5184$

Study purpose: To analyze the effects of co-production and co-creation on value co-creation and on the satisfaction of financial services' consumers.

Methodology/Approach: We used structural equations modeling [SEM] for the empirical assessment of the theoretical model based on SDL: value stems from interaction in co-production or co-creation (Vargo \& Lusch, 2004), and engagement in co-creation and co-production, which have positive effects on value creation (Prahalad \& Ramaswamy, 2000, 2004; Vargo \& Lusch, 2004), and satisfaction (Williams \& Naumann, 2011).

Main findings: The model showed statistical results close to acceptable values (Hair et al., 2009), but not sufficient to affirm that there is an effect of coproduction and cocreation on co-created value and on customer satisfaction. There is correlation between the variables of co-production and co-creation, but the low explanatory power requires adjustments to the model or a review of the constructs.

Theoretical/methodological contributions: We highlight the relationship between co-production and co-creation, since the model extends knowledge about their constructs applied to the financial context, with categories and modeling proposition, by refining the ideas of co-production, co-creation, value cocreation, and perceived value.

Relevance/originality: The study builds parameters for measuring co-created value in co-creation and co-production of services, and shows that customers of financial services co-produce or co-create as an extension of the supplier, not of the customer.

Keywords: Co-creation and Co-production. Value Co-creation. Satisfaction. Financial Services.

\section{How to cite the article:}

Morais, F. R., Pandolfi, E.S. de, \& Sanagioto, L. T. (2020). Does co-creation and co-production create value and customer satisfaction? analysis on the perception of financial institutions' Clients. Brazilian Journal of Marketing, 19(1), 117-140. https://doi.org/10.5585/remark.v19i1.17141.

\footnotetext{
1 (D) Universidade Federal de Rondônia - UNIR, Rondônia, (Brasil). fabiomorais @ unir.br

${ }^{2}$ Faculdade de Educação e Meio Ambiente - FAEMA, Rondônia, (Brasil). edgarpandolfi@gmail.com

3 Faculdades Associadas de Ariquemes - FAAr, Rondônia, (Brasil). luciana.sanagioto@ hotmail.com
} 


\section{Introduction}

Co-creation and co-production of services are a new paradigm in business management literature, where building relational value is evident, as opposed to economic theories of value derived exclusively from the transaction (Galvagno \& Dalli, 2014; Pohlmann \& Kaartemo, 2017). Co-creation is the customer's participation in the collaborative process of producing material or symbolic value (Galvagno \& Dalli, 2014; Gronröos, 2009; Lusch \& Vargo, 2006), while coproduction involves the client in the production of goods and services, by carrying out all or part of it with the supplier (Agrawal \& Rahman, 2015; Ostrom, 1996). This proposition highlights that there is a sequence of consumer needs for establishing relationships and undergoing valuable experiences in economic exchanges (Prahalad \& Ramaswamy, 2004), and suppliers' needs to share the production with customers (Etgar, 2008). Thus, the Service Dominant Logic [SDL] suggests that value results from the interaction between customers and suppliers in the joint production or creation of products and services (Vargo \& Lusch, 2004).

Although literature emphasizes that value and consumer satisfaction in transactions (Williams \& Naumann, 2011) are built through a relationship that is capable of creating valuable experiences in the processes of economic exchanges (Prahalad \& Ramaswamy, 2004), it is necessary to advance knowledge on how and where value is co-created in the processes of coproduction and co-creation of services. There is evidence that the existence of a relationship, in itself, is not sufficient to create value and satisfaction of the co-producer or co-creator customer. SDL suggests that customer's interaction and engagement in co-creation and co-production of goods and services has a positive effect on building the joint valuable experience (Prahalad \& Ramaswamy, 2000, 2004; Vargo \& Lusch, 2004) and on consumer satisfaction (Williams \& Naumann, 2011). Still, the interaction and engagement between customers and suppliers in coproduced or co-created processes improve production efficiency, long-term cost reduction, and quality of the provided services (Alford, 2002; Bendapudi \& Leone, 2003; Gronröos, 2009, 2011; Morais \& Santos, 2015; Ramirez, 1999), in addition to contributing to a higher financial return for organizations (Williams \& Naumann, 2011).

SDL (Vargo \& Lusch, 2004) distinguishes co-production from co-creation through the customer's role in the joint process of value creation. Co-production sees the consumer as a passive agent, and focuses on how the organization can achieve production benefits through consumer's involvement in production, while co-creation classifies the customer as an active agent in his relationship with the organization (Prahalad \& Ramaswamy, 2004 ; Vargo \& Lusch, 2004). Their proposal is a highlight in the management literature and in corporate environment, with an evolutionary trajectory in the areas of value co-creation, integration of resources, brands, and innovation (Pohlmann \& Kaartemo, 2017). However, there are criticisms to SDL, especially 
regarding the metaphorical vision of value co-creation (Gronröos \& Voima, 2013) and the absence of metrics for the empirical sizing of the value that stems from co-creation and co-production practices (Morais \& Santos, 2015). Results of previous research indicate that the scientific community has intensified the assessment of the fundamental ontological and epistemological issues of SDL, such as complex phenomena, generic concepts, democratization processes, and institutionalization practices (Pohlmann \& Kaartemo, 2017).

In this context, our research followed the studies by Ramirez (1999), Vargo and Lusch (2004), Etgar (2008), Gronröos (2009, 2011), and Morais and Santos (2015), and sought to identify the effects of co-creation and co-production on co-created value, and the satisfaction of financial services' users, characterized as an evaluation of institutionalized practices (Pohlmann \& Kaartemo, 2017). The proposition is that service consumers are resource suppliers and participants in value creation (Prahalad \& Ramaswamy, 2004; Vargo \& Lusch, 2004), and co-creation and co-production increase their perceived value and satisfaction. To that end, we assessed the attributes and results of daily interactions in physical agencies or other service environments, in this case financial services, which are determinant for value co-creation and consumer satisfaction (Parasuraman, Zeithaml, \& Berry, 1985). Therefore, we sought to answer the following research question: what are the effects of co-creation and co-production of financial services on the perception of co-created value, and on the satisfaction perceived by consumers?

We chose to examine the financial sector because literature mentions it as a relevant area for co-production and co-creation of services. There is a relationship between co-production and customer loyalty in the financial system (Auh, Bell, McLeod, \& Shih, 2007), and the interaction between the consumer and the financial services' company favors the creation or joint production of consumer goods, enabling joint value creation, according to the particularities of customers (Prahalad \& Ramaswamy, 2004). Studies highlight that financial institutions undergo continuous restructuring of production processes, and emphasize the improvement of the provided services to meet customers' needs (Arasli, Mehtap-Smadi, \& Katircioglu, 2005). Thus, mutually beneficial relationships between consumer and organization allow the exchange of experiences and, consequently, promote value creation, both in co-creation and co-production (Morais \& Santos, 2015).

Ribeiro, Machado and Tinoco (2010) state that customer satisfaction and loyalty increase the profitability of financial institutions, but the elements linked to value co-creation and their influence on satisfaction need to be examined. Thus, the survey sample consisted of bank and credit cooperative customers. Although clients associated to credit cooperatives have distinct attributes from bank customers - besides using the products they have an ownership interest - and for that reason they are more likely to co-create (Duarte, Moreira, Ferraresi, \& Gerhard, 2016), we found 
little evidence in the literature to justify these differences. Therefore, we decided to assess them separately, as for the action of co-creation and co-production, but together regarding the results of perceived value and satisfaction by customers.

\section{The model and hypotheses development}

This study evolved from the theoretical model proposed by Morais and Santos (2015), where the authors show that value co-creation is the element resulting from the interaction and engagement of the customer in the process of joint creation and manufacturing of products and services (Figure 1). The model shows that focus on the supplier and production are on the $\mathbf{x}$ axis, where $\boldsymbol{x}$ is the design matrix of fixed effects, with dimensions $n \times p$. Customer and use/consumption are on the $\mathbf{y}$ axis, where $\mathbf{y}$ is a vector of observable variables, with dimensions $n \times l$. And $z$ derives from value co-creation that results from the interaction between $\boldsymbol{x}$ or $\boldsymbol{y}$, where $z$ is the incidence matrix of random effects (co-variables), with dimensions $\mathbf{n} \times \boldsymbol{q}$. The interaction between $(\mathbf{x})$ and $(\mathbf{y})$ axes may present variation in co-created value at the vertex of the $(z)$ axis, according to the determinants of the interaction, in the economic relationship between customer and supplier (Morais \& Santos, 2015).

Given the theoretical gaps identified, the general purpose of this research was to examine the effect of co-creation and co-production of services (supplier/consumer) on the perception of cocreated value and consumer satisfaction, regarding financial services. Hence, the model shows that the greater the interaction between the customer engaged in the co-creation or co-production process and the supplier, the greater the perceived co-created value (Morais \& Santos, 2015). We add to this empirical analysis the dimension 'consumer satisfaction', to check if co-creation and co-production have an effect on it. 
Figure 1 - Theoretical model of analysis

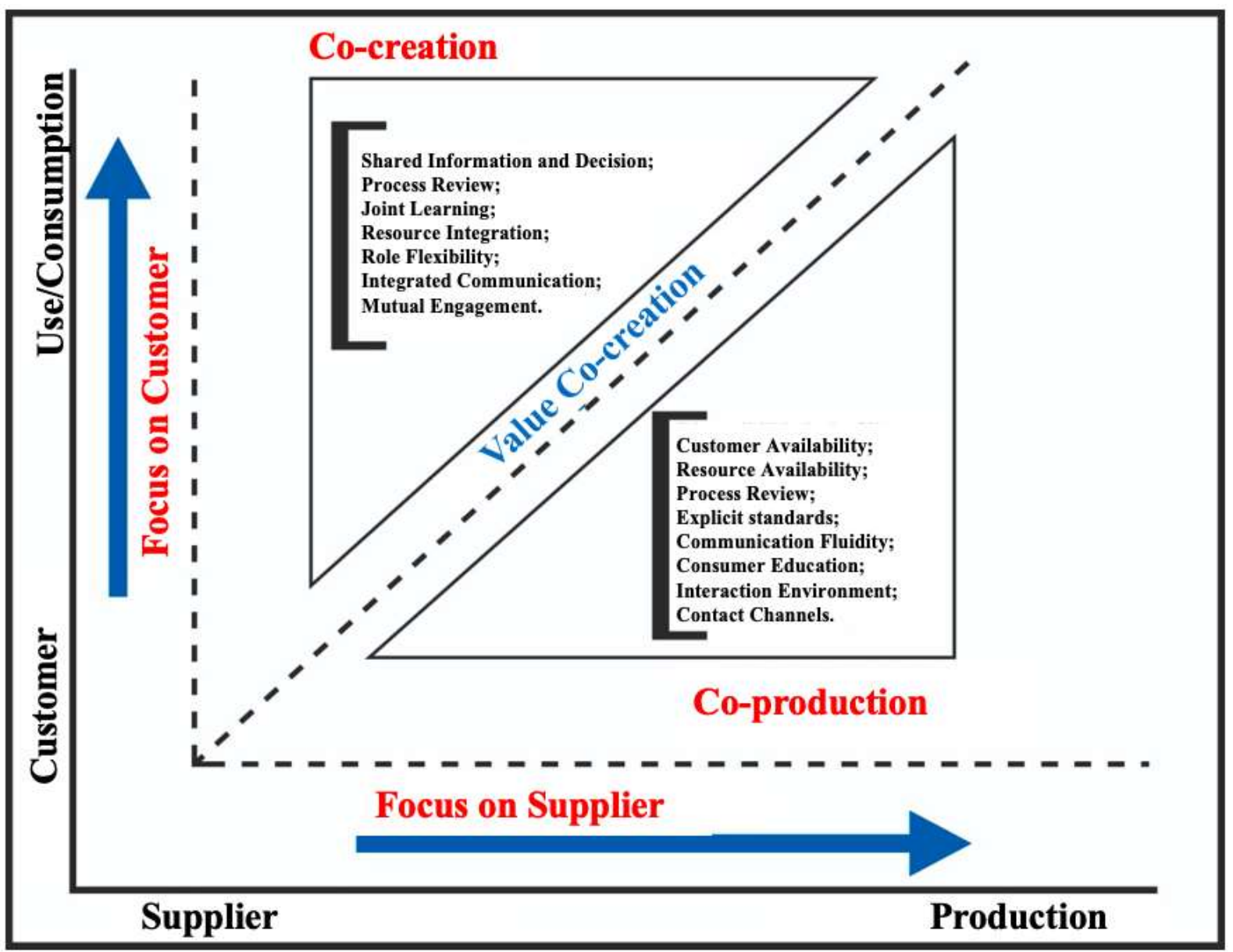

Source: Morais \& Santos (2015, p. 245).

We used structural equations modeling (SEM) as a confirmatory technique, by developing the model before data collection, and the objective was to check if the collected data would confirm or not the theoretical model. SEM is a research method that involves activities and decisions on the theoretical framework, the structural model design, and the measurement model (Hair, Black, Babin, Anderson, \& Tatham, 2009).

SEM allows confirmatory tests of the psychometric structure of measurement scales, in addition to analyzing explanatory relationships among multiple variables, simultaneously, whether these are latent or observed (Hair et al., 2009). Thus, we used SEM to examine more than one dependent variable, where $\mathbf{x}$ influences $\mathbf{y}$ and $\mathbf{y}$ affects $\mathbf{z}$, as services are inherently co-created or co-produced (Chathoth, Altinay, Harrington, Okumus, \& Chan, 2013) in different areas of the interaction between customers and suppliers (Morais \& Santos, 2015). The justification for SEM's use is that we can get causal models compatible with the data, and confirm the alternative that has stronger support from theory. This technique allows the investigation of how well the predictor variables explain the dependent variables, and which of them are more important for explaining the 
model (Hair et al., 2009). To achieve methodological quality with structural equations modeling, we used the parameters established by Bido, Souza, Silva, Godoy and Torres (2012), where they present the appropriate steps for SEM's preparation and use. Next, we present the hypotheses based on the theory and the proposed structural model, with the expected direction of the parameters established for confirmation.

\subsection{Hypotheses development}

Co-creation happens when the customer is part of the process of products and services' creation, developed through the relationship with the organization (Gronröos, 2009). Therefore, it allows different actors to participate and contribute to the creation process in economic exchanges, through shared competences and knowledge (Vargo \& Lusch, 2004), which result in the use and consumption of goods that meet the needs of each party (Grönroos, 2011). On the other hand, coproduction is the customer's participation, within parameters pre-established by the supplier, by doing all or part of the production process in an industry (services/products) (Etgar, 2008; Ostrom, 1996; Ojasalo, 2010).

Different engagement processes and concepts (Morais \& Bonomi, 2015) support coproduction and co-creation, for the access to resources that the involved actors believe to have potential value (Chandler \& Vargo, 2011). The two relationships, although distinct, develop on similar relational platforms, except for the modus operandi of the players involved in building the value result. Co-creation centers its processes on the customer (Gronröos, 2009), while coproduction focuses on the supplier (Ramirez, 1999; Wu, Wang \& Rouyer, 2019); however, management theory, when addressing value creation through both, does not present them as opposing activities.

Co-production carried out to create a certain product or service may lead to the evolution of engagement for co-creation. This can happen through small changes in the production bases of what is co-produced (product or service), as the emergence of new uses for the product, or the co-creation of new processes that favor performance, or still the insertion of some co-produced product/service in new markets co-created with consumers, especially through digital means (Nambisan \& Baron, 2007, 2009).

This reinforces the idea that, although co-production is an activity centered on the supplier (Gronröos, 2011), it may require actions that exceed the joint production act (supplier/consumer) and evolve towards the co-creation of new production processes, and of consumer or utility markets for what is co-produced (Ramirez, 1999; Wu, Wang \& Rouyer, 2019). Therefore, the transition from co-production to co-creation can happen through the expansion of attributions to consumers that participate in joint production processes between suppliers and consumers, in order to expand 
or improve products, processes and markets. The theoretical assumption is that co-production has a positive effect on co-creation (Chandler \& Vargo, 2011). Hence,

H1: The co-production action with suppliers can evolve towards co-creation of services, according to consumers' perception.

In addition, the value experience with co-production and co-creation falls within the relationship sphere (Prahalad \& Ramaswamy, 2004). It is through the interaction of the supplier with the customer, by engaging him in the creation and production process, that the effect on cocreated value takes place (Grönroos \& Voima, 2013; Morais \& Santos, 2015). Co-created value stems from the mutual influence of the players, and can happen directly, in physical areas of contact, or through virtual means, through technological mechanisms for engaging in co-creation and coproduction (Nambisan \& Baron, 2007, 2009).

Payne, Storbacka and Frow (2008) state that the interaction when co-producing or cocreating can create value for the customer. However, co-production classifies the consumer as a passive agent, focusing on the benefits from his involvement in production, while co-creation sees the customer as an active agent in the relationship with the organization (Prahalad \& Ramaswamy, 2004; Vargo \& Lusch, 2004), when acting as a creative part of the service to be offered. Hence, there should be differences in the results of perceived value, when comparing the consumer perception; there are two bases of value co-creation, which can lead to different perceptions of cocreated value (Morais \& Santos, 20015; Vargo \& Lusch, 2004; Gronröos, 2009, 2011).

The context where value is co-created supports the analysis, which stems from the orientation of the process of value creation (multi-level) (Chandler \& Vargo, 2011): horizontal, where co-production coordinates the activities, and vertical, where co-creation requires the integration of partners. This can lead to distinct perceptions of co-created and appropriated value, even if the approaches are based on interaction and relationship (Grönroos, 2011).

There is evidence that the integration of the supplier with the consumer improves the performance of the Product Development Process, with a reduction in time and products more appropriate to the market (Prandelli, Verona, \& Raccagni, 2006). There is also evidence of the positive effects of co-creation on the evaluation of the final product (Yin, Chan, \& Lam, 2012). However, the analysis of the value resulting from the joint action in co-creation is absent from the mentioned studies. The same happens with the action called co-production (Ramirez, 1999; Vargo \& Lush, 2004), whose decision is also to incorporate the consumer skills. According to our research proposal, co-creation and co-production have an impact on consumers' perception regarding cocreated value. The action of participation (co-creation and co-production) builds a sense of 
collaboration and engagement (Thompson \& Malaviya, 2013) that leads the consumer to perceive the added value. Therefore, the act of co-creating or co-producing with the consumer can have an effect on his perception of value. Specifically,

H2a: the co-production of services depends on the production context and consumer engagement, which is why the effect on the perception of co-created value is insignificant in the coproduction of services with low engagement.

$H 2 b$ : the co-creation of services requires stronger engagement with consumers and, therefore, has a positive effect on the perception of co-created value.

In addition, the relational offer and the interaction between the parties have different levels of engagement, since some consumers accept to engage and others do not (Morais \& Santos, 2015). There is still the influence of supplier dimensions that inhibit or facilitate interaction in the relationship with customers. Thus, co-created value differs according to how customers interact and engage.

Co-production can increase customer satisfaction (Prahalad \& Ramaswamy, 2004), but the focus is on the organization's financial performance, with the total or partial transfer of production (Williams \& Naumann, 2011). Co-creation can increase customer satisfaction (Gronröos, 2009), but the focus is on the organization's product, with the total or partial transfer of the good to the consumer (Prandelli et al., 2006; Yin, Chan, \& Lam, 2012). Ramirez (1999) states that consumer perception is determined by experiences; therefore, the way value is co-created can enhance consumer satisfaction (Kauffmann \& Marchetti, 2008). According to Zeithaml, Bitner and Gremler (2006), customer perception is what establishes, or not, satisfaction in services.

Co-creation and co-production processes are different in the way of interaction and in the degree of consumer engagement (Morais \& Santos, 2015; Vargo \& Lusch, 2004; Gronröos, 2009), and the relationship between consumer and supplier can influence the perceived satisfaction, through the assessment of the interactions' level (Shankar, Smith, \& Rangaswamy, 2003). Cocreation is a consumer decision, and results from his own choices to work with the supplier or not (Etgar, 2008), since it can be understood as the sequence of customer activities for value creation (Edvardsson, 1997; Eichentopf, Kleinaltenkamp, \& Van Stiphout, 2011). On the contrary, coproduction is based on organizational interest, although it can create value that results from the customer's experiences of production and consumption (Morais \& Santos, 2015).

Therefore, if the consumer has a value perception within the set of co-creation and coproduction of services, the result of this perception on the offer (costs) and the exchange (benefits) regards the good, and satisfaction becomes a consequence of the perceived value (Zeithaml, 1988; 
Zeithaml et al., 2006). The participation relationship in co-creation and co-production adds value (Morais \& Santos, 2015), and can reflect on future relationship decisions (Thompson \& Malaviya, 2013), according to consumer satisfaction (Zeithaml et al., 2006). The consumer internalizes the experience with the service provider, which can reinforce his perception on the supplier's reliability and integrity (Singh \& Sirdeshmukh, 2000). Hence,

H3: the co-created value during the experience of co-creation and co-production is what determines the consumer perceived satisfaction.

We still highlight that the different levels of consumer engagement (Morais \& Santos, 2015) can change the basis of interaction and, consequently, of satisfaction (Prahalad \& Ramaswamy, 2004). Hence, the types of experience can influence the consumer satisfaction perception, with a different perception of satisfaction levels between co-created or co-produced services.

If co-creation and co-production processes have different levels of interaction and consumer engagement (Morais \& Santos, 2015; Vargo \& Lusch, 2004; Gronröos, 2009), and satisfaction is a consequence of the perceived value (Zeithaml, 1988; Zeithaml et al., 2006), then co-creation and co-production may present different levels of satisfaction in terms of perceived value.

Therefore, for this research, we considered that the consumer who co-creates and the consumer who co-produces have different perceptions of co-created value, and, consequently, of satisfaction. Thus, we developed a hypothesis on the influence of co-creation and co-production on the perception of satisfaction:

H4: co-creation and co-production of services have different impacts on consumer satisfaction, due to differences in the level of consumer engagement.

\section{Methodology}

The target population of this study were customers of banks and credit cooperatives in a Brazilian city at the country's northern region. We chose the field of financial services due to the high involvement of customers in the processes of co-creation (credit cooperatives) and coproduction (banks) of services (Auh et al., 2007; Duarte et al., 2016). The current scenario of the financial segment has the best conditions for a researcher to check and evaluate the choices available to clients, for co-creating and co-producing services, and to identify the effect on the perceived cocreated value. Another aspect considered in the selection of this target population were the conditions for assessing customer satisfaction after co-production or co-creation of services. As 
literature shows, co-creation and co-production result in co-created value for the consumer, and consequently, in greater satisfaction.

The sample consisted of the population of a Brazilian city, and we considered the total population index measured by the Brazilian Institute of Geography and Statistics [IBGE], regarding the 2010 census, which we updated for the year 2017 through annual population estimates. The sample included only individual customers, determined by the type of relationship that requires engagement and control in the process of co-creation and co-production of services.

Two assistants that applied the 7-point Likert scale, both specialists in management, explained the terms 'co-creation' and 'co-production' to the participants. Due to the challenges of selecting a sample of consumers who co-operated in financial services (this was the biggest challenge), it was a convenience, non-probabilistic sample, with 196 individuals. We calculated the sample size based on a finite population, according to the following formula:

Figure 2 - Sample Calculation (Used Formula)

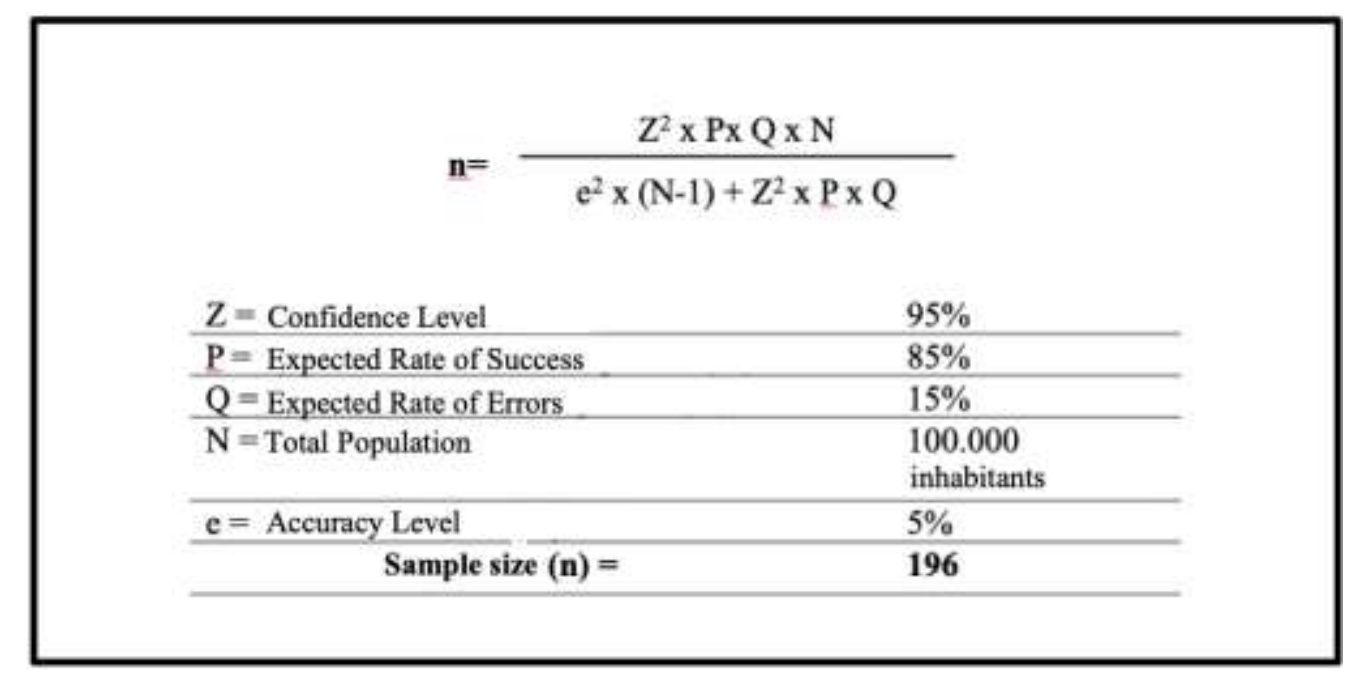

Source: Adapted from McClave, Benson, \& Sincich (2009).

The geographical delimitation aimed to consider a specific population, since the global sample of bank customers would make the research unfeasible. This is one of the limitations of the study, and an opportunity for future research in other regions, as we comment in the final remarks.

To build the scale of measures, we used components of interval scales already validated by Bearden \& Netemeyer (1999), on the following topics. For co-creation: shared information; shared decision; process review; joint learning; resource integration; role flexibility; integrated communication; and mutual participation (engagement). And for co-production: customer availability; resource availability; process review; explicit standards; communication fluidity; consumer education; interaction environment; and contact channels (Morais \& Santos, 2015). 
We used the 7-point Likert scale type to measure the customer's creation and co-production regarding his perception of co-created value and satisfaction. After organizing the scale, we ran a pre-test with 66 people, who were not included in the main sample.

We checked scale reliability through confirmatory factor analysis, using the SPSS 23.0 software to confirm the model fit, as shown in Table 1. As for the adequacy measures of factor analysis, the KMO test (Kaiser-Meyer-Olkin) achieved a value of 0.614 , considered adequate, and statistically significant $(\mathrm{p}<0.01)$. The Barlett's test for sphericity was significant (chi-square $=$ 392.789253; $\mathrm{df}=120 ; \mathrm{p}<0.001$ ), indicating that factor analysis was adequate (Hair et al., 2009).

Therefore, we reinforce that the value for the Bartlett's test for sphericity was satisfactory, that is, $>0.05$, and the KMO criterion had acceptable parameters for correlations $<0.5$. We stress that the indicators of this research showed correlations above 0.5 and dimensions with reliability above 0.7, as mentioned in the literature (Hair et al., 2009). Also, the configuration of the cocreation and co-production factors are arranged according to the factor grouping; finally, we highlight that the model follows its primary version, without adjustments in its variables or attributes, without any exclusion of items, as shown in Table 1.

Table 3 - Analyzed variables

\begin{tabular}{|c|c|c|c|c|}
\hline Construct & Item & Correlation & $\boldsymbol{C F}$ & $\begin{array}{l}\text { Cronbach's } \\
\text { Alpha }\end{array}$ \\
\hline \multirow{8}{*}{ Co-production } & Client availability to co-produce (COP_1) & 0.704 & 0.5 .76 & \multirow{8}{*}{0.728} \\
\hline & Resource availability to co-produce (COP_2) & 0.697 & 0.702 & \\
\hline & $\begin{array}{l}\text { Process review to adjust to co-production } \\
\text { (COP_3) }\end{array}$ & 0.881 & 0.554 & \\
\hline & Explicit norms for co-production (COP_4) & 0.680 & 0.663 & \\
\hline & Communication fluidity (COP_5) & 0.708 & 0.757 & \\
\hline & $\begin{array}{l}\text { Consumer educational basis for co-production } \\
\text { (COP_6) }\end{array}$ & 0.773 & 0.811 & \\
\hline & Feasible environments for interaction (COP_7) & 0.715 & 0.563 & \\
\hline & $\begin{array}{l}\text { Adequacy of communication channels for co- } \\
\text { production (COP_8) }\end{array}$ & 0.714 & 0.706 & \\
\hline \multirow{8}{*}{ Co-creation } & Information sharing in co-creation $\left(\mathrm{COC}_{-} 1\right)$ & 0.670 & 0.801 & \multirow{8}{*}{0.707} \\
\hline & Shared decision on co-creation (COC_2) & 0.642 & 0.719 & \\
\hline & Process review for co-creation (COC_3) & 0.571 & 0.604 & \\
\hline & Joint learning in co-creation (COC_4) & 0.690 & 0.664 & \\
\hline & $\begin{array}{l}\text { Resource integration between parties for co- } \\
\text { creation (COC_5) }\end{array}$ & 0.528 & 0.754 & \\
\hline & Role flexibility in co-creation (COC_6) & 0.624 & 0.841 & \\
\hline & $\begin{array}{l}\text { Integrated communication in co-creation } \\
\left(\mathrm{COC} \_7\right)\end{array}$ & 0.625 & 0.522 & \\
\hline & Mutual participation in co-creation (COC_8) & 0.634 & 0.523 & \\
\hline \multirow[t]{2}{*}{$\begin{array}{l}\text { Perception of } \\
\text { Co-created Value }\end{array}$} & $\begin{array}{l}\text { Perception of co-created value in co-creation } \\
\text { (VCC) }\end{array}$ & 0.530 & 0.686 & \multirow{2}{*}{0.634} \\
\hline & $\begin{array}{l}\text { Perception of co-created value in co- } \\
\text { production }(\mathrm{VCP})\end{array}$ & 0.465 & 0.695 & \\
\hline \multirow{3}{*}{$\begin{array}{l}\text { Perceived } \\
\text { Satisfaction }\end{array}$} & Perceived satisfaction (COP) & 0.554 & 0.554 & \multirow{3}{*}{0.554} \\
\hline & Perceived satisfaction (COC) & 0.554 & 0.554 & \\
\hline & Perceived satisfaction (VC) & 0.554 & 0.554 & \\
\hline
\end{tabular}

Source: Morais \& Santos (2015, p. 245) - Scale adapted from Bearden \& Netemeyer (1999). 


\section{Data analysis}

According to Arbuckle (2006) and Anderson and Gerbing (1988), we used two steps to carry out the SEM analysis procedure with AMOS 24.0. The first stage consisted of quality criteria through Confirmatory Factor Analysis (CFA), to ensure the reliability and validity of data, when testing the causal relationships between the latent variables, in the second stage. Convergent validity showed an average variance extracted with a minimum value above 0.4 and factorial loads above 0.5. Although they were not representative values, they were acceptable for the model conditions. We confirmed discriminant validity between the latent variables, since correlation did not exceed the squared variance extracted (latent variables), and the factorial loads of the items were higher in their respective constructs than in other variables (Table 2).

Table 4 - Discriminant validity of latent variables

\begin{tabular}{|l|c|c|c|c|}
\hline & Co-creation & Co-production & Perceived Co-created Value & Satisfaction Perception \\
\hline Co-creation & 0.52 & & & \\
\hline Co-production & - & 0.50 & & \\
\hline Perceived Co-created Value & - & - & 0.58 & \\
\hline Satisfaction Perception & - & - & - & 0.54 \\
\hline
\end{tabular}

We carried out the goodness-of-fit [GOF] through multiple indicators: $\chi^{2}$ (chi-square), $\chi^{2} / \mathrm{df}$ (chi-square per degrees of freedom), CFI (comparative fit index), GFI (model's good fit index), TLI (Tucker Lewis index), and RMSEA (root mean square error of approximation). According to Hair et al. (2009), the model fit is good when the indexes are $\geq 0.90, \chi^{2} / \mathrm{df}$ between 2 and 5 , and RMSEAs $\leq 0.08$. 
Figure 3 - Fit model

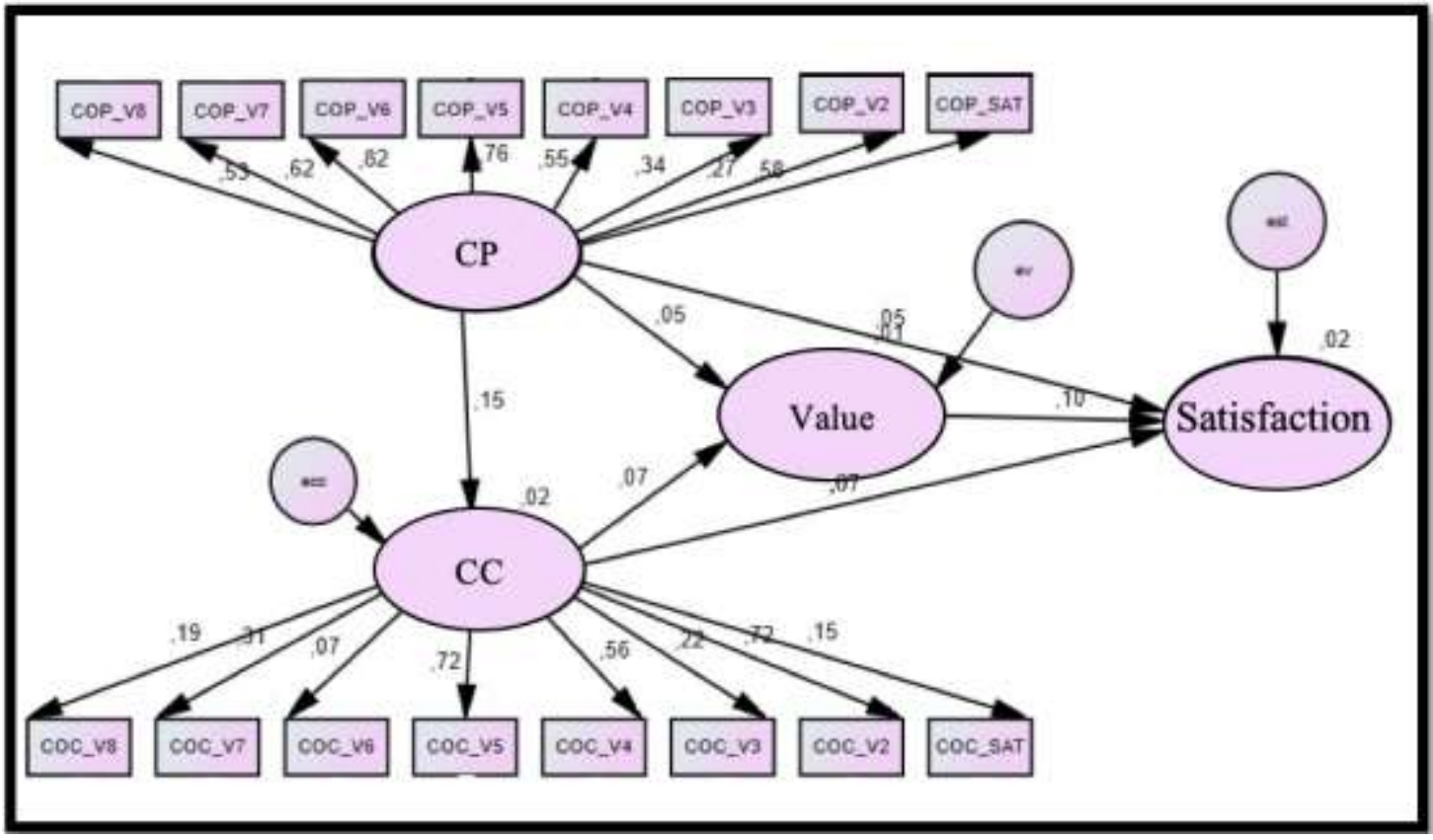

This model showed $\chi^{2}(211.642), \chi^{2} / \mathrm{df}(211.642 / 103=2.055)$, CFI $(0.625)$, TLI $(0.550)$ and RMSEA (0.153), and all statistics are close to acceptable values (Hair et al., 2009). However, it is not sufficient to assert that there is an effect of co-production and co-creation on co-created value and customer satisfaction. Pearson's correlation was not significant $(\mathrm{p}<0.01)$ between the variables co-production and co-creation, and co-created value and co-produced value. According to the graphic model, the only possible statement is on the existence of correlation between the variables of co-production and co-creation, which have significant explanatory capacity for the joint action of production or creation (Morais \& Santos, 2015). The low explanatory power of co-production and co-creation on co-created or co-produced value and customer satisfaction may indicate the need to adjust the model or review the constructs for co-created value.

The estimates of standardized coefficients show that the relationship between co-production and the co-produced value $(\beta=0.05 ; \mathrm{p}<0.01)$, and between co-production and satisfaction $(\beta=$ $0.05 ; \mathrm{p}<0.01)$ have a low explanatory capacity. Similarly, the estimates of the standardized coefficients show that there is also a low capacity for explanation between co-creation and cocreated value $(\beta=0.07 ; \mathrm{p}<0.01)$, and between co-creation and satisfaction $(\beta=0.07 ; \mathrm{p}<0,01)$. Consequently, estimates of standardized coefficients show that the path between co-creation value and satisfaction $(\beta=0.10 ; \mathrm{p}<0.01)$ is also not representative. Thus, results showed that the structural model did not reach a satisfactory goodness of fit, where $\chi^{2}(211.642), \chi^{2} / \mathrm{df}(211.642 / 103=2.055)$, CFI (0.625), TLI (0.505) and RMSEA (0.153) show statistics close to acceptable values (Hair et al., 2009). The results indicate that the only supported relationship is between co-production and co- 
creation $(\beta=173, p=0.01)$, which sustains SDL theory, that services are always co-produced or co-created.

In view of these data, the model was not satisfactory regarding its theoretical elements. It is necessary to consider the limitations of the SEM model under analysis, since there are elements of the research itself that may have contributed to these results. The sample was taken from a single Brazilian city, in the outskirts of the country, and comprised only participants to whom we had access; this can be a limiting factor, and if the research were replicated with a sample selected at random, with a greater regional diversity (national representation), it could lead to different results.

Another important element that adds to the limitations is the context of services that we examined (banks and credit cooperatives). According to theory, services are always co-created or co-produced, but the intensity (level) of the service and the type of consumer needs can change the engagement and, consequently, the perception of value and satisfaction resulting from co-creation and coproduction. Other service sectors, such as health and education, can lead to different answers with a higher impact, due to higher involvement in the co-creation and co-production processes.

Although the theory that we followed emphasizes this theoretical model, and there are similar or complementary results in other studies, the level of acceptability of the statistical indicators raises doubts as to the ideal model. At this point, it is worth mentioning the contribution of one of the paper evaluators, who highlighted the theoretical differences evidenced in our study regarding customers associated to credit cooperatives, because there is a theoretical discussion on the existence of attributes that distinguish them from bank customers. This finding will have implications for SEM, because if there were a distinction in the audience characteristics, the separate analysis of co-creation and co-production, followed by a comparison between them, would bring more reliability and precision to this topic.

\section{Discussion}

For the analysis of results, it is necessary to resume some elements already discussed in the theoretical framework, in order to explain and support our contribution to the existing theory. For approximately fifteen years, management theory has built and discussed models for value cocreation, with the purpose of representing the construction of joint value and measuring co-created value in business relationships. As examples previously mentioned, the model proposed by Prahalad and Ramaswamy (2004) focuses on the environment that stimulates the interaction between the consumer and the supplier, through the DART Model (Dialogue, Access, Risk Assessment, Transparency), which assumes the combination of these four pillars for the engagement of consumers in the company's processes. If these pillars are not used together, they become just facilitators in the process of co-production/co-creation, without any guarantee of co-created value. 
As a complement to Prahalad and Ramaswamy's model (2004), the co-creation model of brand relationship experience, proposed by Payne et al. (2008), assumes the comprehension of the value process (what/how?) in order to understand what is co-created value, and what makes its construction easy. This conception of a model of co-creation value is based on a set of tools used in processes, activities and tasks, through procedures, mechanisms and interactions; if supported by value propositions, they become capable of generating co-created value results. Therefore, customer is the value co-creator, and value only emerges through the use or through a lived and perceived experience.

Finally, the current value model, proposed by Grönroos (2011), is similar to the previous models, as it believes that value co-creation stems from the joint process of developing a service/product, but is different regarding the value co-creation process. The author argues that the consumer carries out the service/product development consciously, through his involvement in the production/creation, but as for co-created value, in general, he perceives it unconsciously, during service provision or by using the product. Thus, value co-creation depends on the perception of value that emerges from the use or under specific conditions, when the consumer is involved in the production/creation (design, development, making, part of the process). Therefore, it is important that companies absorb the needs and expectations of consumers, in order to interact and influence them in value co-creation, since believing that the consumer is always a value co-creator can be a misconception about his role in value co-creation.

However, we stress that these previous models aimed to present theoretical evidence on what is value co-creation and how it happens through consumers' engagement (Prahalad \& Ramaswamy, 2004), in the joint production by company and consumers, in their lived and perceived experience (Payne et al., 2008), and in the specific context of use (Grönroos, 2011). We developed our model with the purpose of suggesting a theoretical pattern for measuring value co-creation (Morais \& Santos, 2015). Thus, our first contribution regards the conceptual limits that make up and sustain the elements that shape the construct 'coproduction' or the construct 'co-creation'.

The coproduction dimension (construct 1) is made of eight categories (observed variables): customer availability, resource availability, process review, explicit rules, communication fluidity, consumer education, interaction environment, and contact channels. In turn, the co-creation dimension (construct 2) is composed of seven categories (observed variables): shared information and decision, process review, joint learning, resource integration, role flexibility, integrated communication, and mutual engagement.

In this context, there is sufficient statistical evidence to say that co-creation and coproduction are constructs that consists of the variables mentioned above, according to the data factor grouping (Cronbach's $\alpha$ ). In addition, we can state that the co-production action with suppliers can 
evolve to co-creation of services (hypothesis $\mathrm{H} 1$ ), according to consumers' perception $\left(\mathrm{R}^{2} 0.15\right)$. Co-production, as the theory highlights, may require the increase of co-creation actions, through the change of the consumer's role in the co-production process. Hence, it changes the role of the coproducer consumer, and extends it to participate in the design of new processes, or new improvements in the usefulness of the product/service or of markets.

Our findings bring a specific theoretical contribution to the organization of the variables mentioned in the existing theoretical framework, according to the significance test of correlation. We can say that there is correlation between the variables that compose co-production and cocreation, and they form the aforementioned analysis constructs. In addition, we identified that coproduction can evolve to more consistent relationships, when the co-producer consumer starts to incorporate the role of co-creator in a single process of value co-creation for the interested parties.

We also observed that although the effects for co-created value and consumer satisfaction are statistically significant in our model $(\mathrm{p}<0.05)$, it has a low explanatory capacity for co-creation $\left(\mathrm{R}^{2}\right.$ 0.05) and co-production $\left(\mathrm{R}^{2} 0.07\right)$; this may be due to the type of engagement and value perception of the chosen consumers (Grönroos, 2011). From this theoretical proposition, hypotheses "H2a" and "H2b" emerge, and the study confirms that there are differences in the ability to explain co-created value by co-production (0.05), and co-created value by co-creation ( 0.07$)$, although not significant (only $2 \%$ difference in explaining results).

The low difference and representation of co-created value with co-production and cocreation may relate to the context, which seems to be of low engagement for our sample. This is another issue to discuss in the suggestions for future research, since, if the perception of co-created value in co-production of services is dependent on the production context and consumers' engagement, there should be a larger distance of the explanatory capacity from co-created value in co-creation processes. Other service contexts, such as education and health, may require a stronger consumer engagement. Therefore, according to the perception of the consumer that participates in the co-creation and co-production processes, although there are statistical differences in the perception of co-created value ( $\mathrm{p}$-value $<0.05$ ), in the context of this study the results are not sufficient to state that there is a distinction in value that stem from co-production and co-creation.

Finally, we highlight that co-created value can mediate the relationship between co-creation and co-production, although we got an explanation of medium statistical significance. Consumer satisfaction can be explained by value co-creation $\left(\mathrm{R}^{2} 0.10\right)$, but the percentage of explanation does not seem to be significant for explaining consumer satisfaction, although it is close to the average for the social sciences field (Ringle, Silva, \& Bido, 2015). However, what in advance seems to be a limitation of the study, allows drawing analyses on the typologies of respondents and the business context. The level or intensity of services changes engagement and value perception, as in the case 
of banks and credit cooperatives, where services have substitutes in brands and business models and, probably, there is less value in the use or in the lived and perceived experience. Therefore, the more essential is the service (rare or non-substitutable), the greater will be its ability to explain cocreated value (Payne et al., 2008).

Thus, this model contributes to broaden the understanding of how value co-creation occurs in co-production and co-creation of services, especially by identifying that co-production and cocreation depend on additional items in the consumers' sphere, so that the company can engage them in unique or non-substitutable offers, thus increasing the perceived value. Furthermore, by testing the existing theoretical framework in a theoretical-empirical analysis (Morais \& Santos, 2015), we extended the dialogue on co-production and co-creation, to achieve value co-creation.

\section{Final remarks}

The main purpose of this study was to identify the effects of co-creation and co-production on value co-created and customer satisfaction. The results showed that there is no significant relationship between co-production and co-creation, and between co-created value and customer satisfaction. They suggest that the theoretical and practical arguments for co-production and cocreation with consumers need a review as to their objectives (Gronröos, 2009). Some potential explanations for these results are: (1) sample bias, given that it addresses a single region; (2) coproduction is a supplier condition, and the individual customer is a production instrument and a means for reducing supplier's costs; therefore, he may not perceive value; (3) managing co-creation with the customer is incipient in the $\mathrm{B} 2 \mathrm{C}$ market, especially in the credit cooperatives surveyed; hence there is no perceived value; and, (4) if satisfaction stems from value, we cannot speak of satisfaction where there is no perceived value.

Thereby, although these elements deal with the research own set of doubts, we can say that the concept in the management literature is also uncertain, regarding co-production and co-creation for building co-created value. These dilemmas stem from a partial or unidimensional framework; hence, from a theoretical perspective, this study contributes to develop measures necessary to confirm that services are always co-produced or co-created, although co-creation and co-production, by themselves, do not generate co-created value. Another contribution was to identify that different models used for measuring co-created value depend on a conceptual perspective of value for the results of effect, since there are differences compared to other models proposed by the management literature on co-created value, and from the conceptual elements of building value with coproduction and co-creation. Finally, we stress that the dependent variable 'consumer satisfaction' is statistically little explained by co-production $\left(\mathrm{R}^{2} 0.7\right)$ and co-creation $\left(\mathrm{R}^{2} 0.5\right)$, when separate; therefore, the small capacity to explain these variables shows that co-creation and co-production, 
by themselves, do not lead to customer satisfaction. Regarding co-created value $\left(R^{2} 0.10\right)$, there are limitations to making inferences about the explanatory capacity for satisfaction, since statistics on co-created value have low significance as a result of co-production $\left(\mathrm{R}^{2} 0.05\right)$ and co-creation $\left(\mathrm{R}^{2}\right.$ 0.07). Therefore, the observed differences between theory and results may stem from the theoretical cut-off of the model itself, and not from the ability of co-created value to create or explain consumer satisfaction.

The managerial contributions of this study point to a greater attention from managers to the administration of co-production and co-creation. Theory highlights that co-created value in cocreation and co-production stems from interaction and engagement with the customer. However, although co-creation and co-production are significant, these actions have no significance for perceived value or satisfaction. This may indicate, for our sample, that customers of financial services co-produce or co-create as an extension of the supplier and not of the customer. As value depends on the context and on the contingencies of actors, resources and relationship, in specific and unique situations (Prahalad \& Ramaswamy, 2004; Edvardson, Tronvoll, \& Gruber, 2011), the context of this study needs a larger theoretical investigation and managerial attention, to identify the gaps of the model that regard the lack of significance for co-created value and for customer satisfaction.

The theory is not yet consolidated, and the built models show limitations, mainly regarding the completeness of the constructs and answers to contexts and macro-environments (Leroy, Cova, $\&$ Salle, 2012; Morais \& Santos, 2015). Thus, as a proposition for future research, we suggest testing new theoretical classes and new models, which may contribute to organize and extend the categories proposed in this model, and the production of other metrics for data collection and empirical tests. We have also found theoretical information that indicates that customers associated with credit cooperatives have different attributes from those of traditional banks; if this is true, when analyzed separately and compared, there will be differences in value co-creation, and this checking could bring new analyses in future research. Finally, we reinforce Morais and Santos (2015) proposition on studies that could refine the ideas of co-production, co-creation, value co-creation, and perceived value, under other theoretical bases besides the Service Dominant Logic (SDL).

\section{References}

Agrawal, A. K., \& Rahman, Z. (2015). Roles and resource contributions of customers in value cocreation. International Strategic Management Review, 3(1-2), 144-160.

doi:10.1016/j.ism.2015.03.001

Alford, J. (2002). Why do public-sector clients coproduce? Toward a contingency theory. Administration \& Society, 34(1), 32-56. doi:10.1177/0095399702034001004 
Anderson, J. C., \& Gerbing, D. W. (1988). Structural equation modeling in practice: A review and recommended two-step approach. Psychological Bulletin, 103(3), 411-423.

Arasli, H., Mehtap-Smadi, S., \& Turan Katircioglu, S. (2005). Customer service quality in the Greek Cypriot banking industry. Managing Service Quality: An International Journal, 15(1), 41-56. doi:10.1108/09604520510575254

Arbuckle, J. L. (2006). Amos (version 7.0) [computer program]. Chicago: SPSS.

Auh, S., Bell, S. J., McLeod, C. S., \& Shih, E. (2007). Co-production and customer loyalty in financial services. Journal of Retailing, 83(3), 359-370. doi:10.1016/j.jretai.2007.03.001

Bearden, W. O., \& Netemeyer, R. G. (1999). Handbook of marketing scales: Multi-item measures for marketing and consumer behavior research (2nd ed.). Thousand Oaks, CA: Sage Publications.

Bendapudi, N., \& Leone, R. P. (2003). Psychological implications of customer participation in coproduction. Journal of Marketing, 67(1), 14-28. doi:10.1509/jmkg.67.1.14.18592

Bido, D. S., Souza, C. A., Silva, D., Godoy, A. S., \& Torres, R. R. (2012). Qualidade do Relato dos Procedimentos Metodológicos em Periódicos Nacionais na Área de Administração de Empresas: o caso da modelagem em equações estruturais nos periódicos nacionais entre $2001 \mathrm{e}$ 2010. Organizações \& Sociedade, 19(60), 125-144. doi:10.1590/S1984-92302012000100008

Chandler, J. D., \& Vargo, S. L. (2011). Contextualization and value-in-context: How context frames exchange. Marketing Theory, 11(1), 35-49. doi:10.1177/1470593110393713

Chathoth, P., Altinay, L., Harrington, R. J., Okumus, F., \& Chan, E. S. (2013). Co-production versus co-creation: A process based continuum in the hotel service context. International Journal of Hospitality Management, 32, 11-20. doi:10.1016/j.ijhm.2012.03.009

Duarte, A. F., Moreira, V. R., Ferraresi, A. A., \& Gerhard, A. (2016). Evaluating credit union members' perception of service quality through service innovation. INMR - Innovation \& Management Review, 13(4), 242-250. doi:10.1016/j.rai.2016.06.007

Edvardsson, B. (1997). Quality in new service development: Key concepts and a frame of reference. International Journal of Production Economics, 52(1-2), 31-46. doi:10.1016/S0925$\underline{\text { 5273(97) } 80765-7}$

Edvardsson, B., Tronvoll, B., \& Gruber, T. (2011). Expanding understanding of service exchange and value co-creation: a social construction approach. Journal of the Academy of Marketing Science, 39(2), 327-339. doi:10.1007/s11747-010-0200-y

Eichentopf, T., Kleinaltenkamp, M., \& Van Stiphout, J. (2011). Modelling customer process activities in interactive value creation. Journal of Service Management, 22(5), 650-663. doi:10.1108/09564231111174997

Etgar, M. (2008). A descriptive model of the consumer co-production process. Journal of the Academy of Marketing Science, 36(1), 97-108. doi:10.1007/s11747-007-0061-1

Galvagno, M., \& Dalli, D. (2014). Theory of value co-creation: a systematic literature review. Managing Service Quality, 24(6), 643-683. doi:10.1108/MSQ-09-2013-0187 
Grönroos, C. (2009). Towards service logic: The unique contribution of value co-creation. Hanken School of Economics, CERS - Centre for Relationship Marketing and Service Management. Retrieved from http://www.hanken.fi/public.

Grönroos, C. (2011). A service perspective on business relationships: The value creation, interaction and marketing interface. Industrial Marketing Management, 40(2), 240-247. doi:10.1016/j.indmarman.2010.06.036

Grönroos, C., \& Voima, P. (2013). Critical service logic: making sense of value creation and cocreation. Journal of the Academy of Marketing Science, 41(2), 133-150. doi:10.1007/s11747$\underline{012-0308-3}$

Hair, J. F., Black, W. C., Babin, B. J., Anderson, R. E., \& Tatham, R. L. (2009). Análise multivariada de dados. Porto Alegre: Bookman Editora.

Kauffmann, S., \& Marchetti, R. Z. (2008). Canais de atendimento bancário e satisfação do cliente: um estudo em bancos de varejo. In Anais do $3^{\circ}$ Encontro de Marketing da Associação Nacional de Pós-Graduação e Pesquisa em Administração [ANPAD], Curitiba, PR.

Leroy, J., Cova, B., \& Salle, R. (2012). The Value Co-Creation Concept: Mixing Up Apples and Oranges? In 28th Annual Industrial Marketing and Purchasing Group Conference, IMP Group, Rome, Italy. Retrieved from https://hal.univ-reunion.fr/hal-01655560/document

Lusch, R. F., \& Vargo, S. L. (2006). Service-dominant logic: reactions, reflections and refinements. Marketing Theory, 6(3), 281-288. doi:10.1177/1470593106066781

McClave, J. T., Benson, P. G., \& Sincich, T. (2009). Estatística para Administração e Economia (10a ed.). São Paulo: Pearson Prentice Hall.

Morais, F. R., \& Santos, J. B. (2015). Refinando os conceitos de cocriação e coprodução: Resultados de uma crítica da literatura. Revista Economia \& Gestão, 15(40), 224-250. doi:10.5752/P.1984-6606.2015v15n40p224

Nambisan, S., \& Baron, R. A. (2007). Interactions in virtual customer environments: Implications for product support and customer relationship management. Journal of Interactive Marketing, 21(2), 42-62. doi:10.1002/dir.20077

Nambisan, S., \& Baron, R. A. (2009). Virtual customer environments: testing a model of voluntary participation in value co-creation activities. Journal of Product Innovation Management, 26(4), 388-406. doi:10.1111/j.1540-5885.2009.00667.x

Ojasalo, K. (2010). The shift from co-production in services to value co-creation. The Business Review, Cambridge, 16(1), 171-177.

Ostrom, E. (1996). Crossing the great divide: coproduction, synergy, and development. World Development, 24(6), 1073-1087. doi:10.1016/0305-750X(96)00023-X

Parasuraman, A., Zeithaml, V. A., \& Berry, L. L. (1985). A conceptual model of service quality and its implications for future research. Journal of Marketing, 49(4), 41-50.

doi:/10.1177/002224298504900403 
Payne, A. F., Storbacka, K., \& Frow, P. (2008). Managing the co-creation of value. Journal of the Academy of Marketing Science, 36(1), 83-96. doi:10.1007/s11747-007-0070-0

Pohlmann, A., \& Kaartemo, V. (2017). Research trajectories of Service-Dominant Logic: Emergent themes of a unifying paradigm in business and management. Industrial Marketing Management, 63, 53-68. doi:10.1016/j.indmarman.2017.01.001

Prahalad, C. K., \& Ramaswamy, V. (2000). Co-opting customer competence. Harvard Business Review, 78(1), 79-90.

Prahalad, C. K., \& Ramaswamy, V. (2004). Co-creation experiences: The next practice in value creation. Journal of Interactive Marketing, 18(3), 5-14. doi:10.1002/dir.20015

Prandelli, E., Verona, G., \& Raccagni, D. (2006). Diffusion of web-based product innovation. California Management Review, 48(4), 109-135. doi:10.2307/41166363

Ramirez, R. (1999). Value co-production: intellectual origins and implications for practice and research. Strategic Management Journal, 20(1), 49-65. doi:10.1002/(SICI)10970266(199901)20:1<49::AID-SMJ20>3.0.CO;2-2

Ribeiro, J. L. D., Machado, C. O., \& Tinoco, M. A. C. (2010). Determinantes da satisfação e atributos da qualidade em serviços bancários. Gestão \& Produção, 17(4), 775-790. doi:10.1590/S0104-530X2010000400011

Shankar, V., Smith, A. K., \& Rangaswamy, A. (2003). Customer satisfaction and loyalty in online and offline environments. International Journal of Research in Marketing, 20(2), 153-175. doi:10.1016/S0167-8116(03)00016-8

Singh, J., \& Sirdeshmukh, D. (2000). Agency and trust mechanisms in consumer satisfaction and loyalty judgments. Journal of the Academy of Marketing Science, 28(1), 150-167. doi:10.1177/0092070300281014

Thompson, D. V., \& Malaviya, P. (2013). Consumer-generated ads: does awareness of advertising co-creation help or hurt persuasion? Journal of Marketing, 77(3), 33-47.

doi:10.1509/jm.11.0403

Vargo, S. L., \& Lusch, R. F. (2004). Evolving to a new dominant logic for marketing. Journal of Marketing, 68(1), 1-17. doi:10.1509/jmkg.68.1.1.24036

Williams, P., \& Naumann, E. (2011). Customer satisfaction and business performance: a firmlevel analysis. Journal of Services Marketing, 25(1), 20-32. doi:10.1108/08876041111107032

Wu, L. W., Wang, C. Y., \& Rouyer, E. (2019). The opportunity and challenge of trust and decision-making uncertainty. International Journal of Bank Marketing, 38(1), 199-218. doi:10.1108/IJBM-02-2019-0061

Yim, C. K., Chan, K. W., \& Lam, S. S. (2012). Do customers and employees enjoy service participation? Synergistic effects of self- and other-efficacy. Journal of Marketing, 76(6), 121140. doi:10.1509/jm.11.0205

Zeithaml, V. A. (1988). Consumer perceptions of price, quality, and value: a means-end model and synthesis of evidence. The Journal of Marketing, 52(3), 2-22.

doi: $10.1177 / 002224298805200302$ 
Zeithaml, V., Bitner, M. J., \& Gremler, D. (2006). Services marketing: Integrating customer focus across the firm. Boston, MA: McGraw-Hill/Irwin. 
ANNEX I - Instrument for data collection IDENTIFICATION:

\section{a) Age:}

b) Gender: ( ) M ( ) F

c) Education level

( ) elementary school incomplete ( ) elementary school complete ( ) high school incomplete ( ) high school complete ( ) higher education incomplete ( ) higher education complete

d) How long have you been a member of a credit cooperative or a Bank client, regarding your specific case? For

years.

\section{CO-PRODUCTION OF BANK SERVICES}

Mark the number that best represents your relationship with the credit cooperative or bank, according to your situation (1 for DO NOT AGREE, and 7 for TOTALLY AGREE):

1. I am always available to do my own bank services, that is, I care for producing at my bank's Automated Teller Machine (ATM) and meet my own needs in an independent and self-sufficient way.

\begin{tabular}{lllllll}
\hline 1 & 2 & 3 & 4 & 5 & 6 & 7
\end{tabular}

2. I always have resources available to coproduce with my bank, such as electronic devices compatible with the bank (computers, tablets and smartphones).

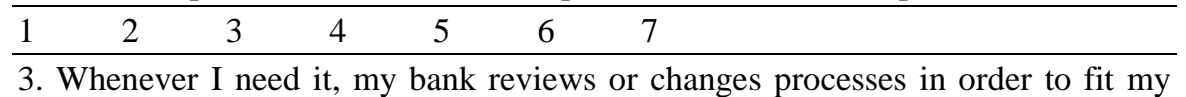
3. Whenever I need it, my bank reviews
demands for self-service and production.

\begin{tabular}{lllllll}
\hline 1 & 2 & 3 & 4 & 5 & 6 & 7
\end{tabular}

4. My bank presents and explains all the rules and tutorials necessary to carry out my own banking service, always clearly and according to what is best for me.

\begin{tabular}{lllllll}
\hline 1 & 2 & 3 & 4 & 5 & 6 & 7
\end{tabular}

5. In my bank, communication is fluid and there are several channels for direct contact with the customer. I am in constant and direct contact, as there are no barriers in communication between the bank and me.

\begin{tabular}{lllllll}
\hline 1 & 2 & 3 & 4 & 5 & 6 & 7
\end{tabular}

6. My bank provides the necessary resources so that I can carry out my services and have a solid banking education as a consumer and producer of my own service.

\section{$\begin{array}{lllllll}1 & 2 & 3 & 4 & 5 & 6 & 7\end{array}$}

7. In my bank, there are feasible environments for interaction, with flexible communication, service performance, with the possibility for me to carry out different actions for the self-production of my banking services.

\begin{tabular}{lllllll}
\hline 1 & 2 & 3 & 4 & 5 & 6 & 7 \\
\hline 8 . My bank has adequate & communication channels for co-production, which
\end{tabular} facilitates the use of the available resources to perform my own services.

$$
\begin{array}{lllllll}
\hline 1 & 2 & 3 & 4 & 5 & 6 & 7
\end{array}
$$

\section{CO-CREATION - PRODUCT/SERVICE IN A CREDIT COOPERATIVE}

1. The credit cooperative shares highly reliable information.

\begin{tabular}{lllllll}
\hline 1 & 2 & 3 & 4 & 5 & 6 & 7 \\
\hline 2. & I try to participate in all activities of the credit cooperative, including meetings
\end{tabular} and decisions. I see the credit cooperative as my home/company, and not as a bank. \begin{tabular}{lllllll}
\hline 1 & 2 & 3 & 4 & 5 & 6 & 7
\end{tabular}

3. I use several services of the credit cooperative, as I helped to plan them in order to meet my needs, since banks would not do the same

$\begin{array}{lllllll}1 & 2 & 3 & 4 & 5 & 6 & 7\end{array}$

4.. Whenever I participate in the services offered by the credit cooperative, there are new learnings, because they listen when I talk about my needs and they explain what is best for me. So we learn together.

\begin{tabular}{lllllll}
\hline 1 & 2 & 3 & 4 & 5 & 6 & 7
\end{tabular}

5. I put resources in the credit cooperative and use the available resources. This happens because the integrated resources are ours.

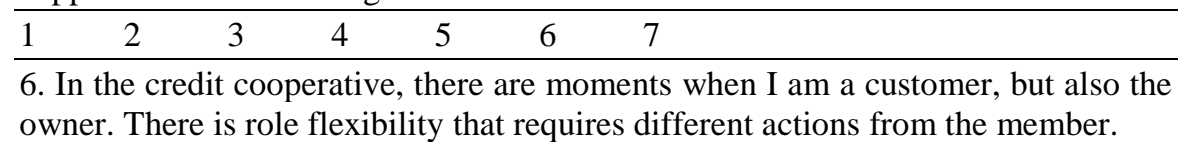
owner. There is role flexibility that requires different actions from the member.

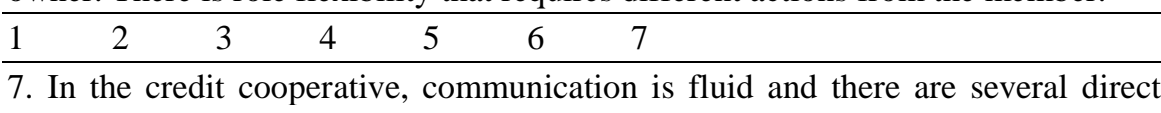
channels for contact with the member. We are in constant contact and there are no barriers to communication

\begin{tabular}{lllllll}
\hline 1 & 2 & 3 & 4 & 5 & 6 & 7
\end{tabular}

8 . We are all one. In the credit cooperative, our biggest good is mutual engagement, and "I" does not exist. The achieved results are for us.

\begin{tabular}{lllllll}
\hline 1 & 2 & 3 & 4 & 5 & 6 & 7
\end{tabular} 


\section{PERCEPTION OF CO-CREATED VALUE}

9. Regarding the services provided by my bank and co-produced by me, I realize that this creates joint value that stems from the type of relationship. My bank adds mutual value when I co-produce my services.

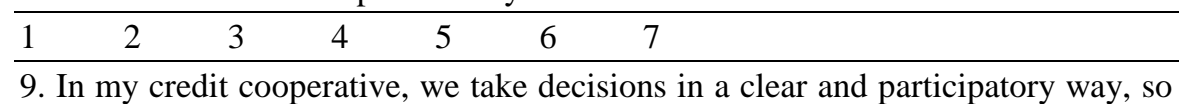
that they suit the member and ensure participation in the creation of products and services, which generates joint value resulting from this type of relationship, by creating products and services together.

\begin{tabular}{lllllll}
\hline 1 & 2 & 3 & 4 & 5 & 6 & 7
\end{tabular}

\section{SATISFACTION PERCEPTION}

10. About your relationship with the credit cooperative, mark the grade that best represents your satisfaction.

\begin{tabular}{lllllll}
\hline 1 & 2 & 3 & 4 & 5 & 6 & 7
\end{tabular}

11. About your relationship with the bank, mark the grade that best represents your satisfaction.

\begin{tabular}{lllllll}
\hline 1 & 2 & 3 & 4 & 5 & 6 & 7 \\
\hline 12. & About your satisfaction that results from the perceived value of the bank or the
\end{tabular} cooperative, mark the grade that best represents your satisfaction.

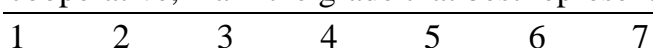

